U. S. DEPARTMENT OF COMMERCE

W, AVERELL HARRIMAN, Secretary

NATIONAL BUREAU OF STANDARDS

E. U. CONDON, Director

\title{
STANDARD STOCK PONDEROSA PINE DOORS

\author{
(SECOND EDITION)
}

\section{COMMERCIAL STANDARD CS120-46}

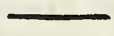

Effective Date for New Production From October 1, 1946

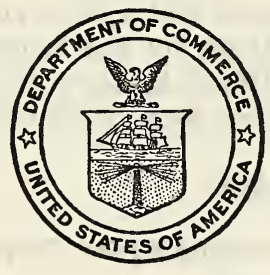

\section{A RECORDED VOLUNTARY STANDARD OF THE TRADE}

UNITED STATES

GOVERNMENT PRINTING OFFICE

WASHINGTON : 1946

For sale by the Superintendent of Documents, U. S. Government Printing Office Washington 25, D. C. - Price 10 cents 


\title{
P R O M U L G A T I N
}

of

COMMERCIAL STANDARD CS120-46

for

\section{STANDARD STOCK PONDEROSA PINE DOORS}

\author{
(Second Edition)
}

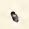

On January 29, 1944, at the instance of the National Door manufacturers Association, a proposed commercial standard for standard stock ponderosa pine doors was submitted to manufacturers, testing laboratories, distributors, and consumer organizations for comment. Following adjustment in the light of the comment, the recommended commercial standard was subsequently accepted in writing by the trade and published as Commercial Standard CS120-44.

A recommended revision, approved by the Standing Committee, was circulated on July 17,1946 , to the trade for written acceptance. Those concerned have since accepted and approved the revised standard, as shown herein, for promulgation by the United States Department of Commerce through the National Bureau of Standards.

The standard is effective for new production from October 1, 1946.

Promulgation recommended.

F. W. Reynolds, Acting Chief, Division of Trade Standards.

Promulgated.

E. U. Condon,

Director, National Bureau of Standards.

Promulgation approved.

W. Averell Harriman, Secretary of Commerce.

Project Manager: J. W. Medley, Division of Trade Standards.

Technical Adviser: V. B. Phelan, Division of Codes and Specifications. 


\section{STANDARD STOCK PONDEROSA PINE DOORS ${ }^{1}$}

\section{COMMERCIAL STANDARD CS120-46}

\section{PURPOSE}

1. The purpose of this commercial standard is to establish standard specifications and sizes for ponderosa pine, standard stock doors to guide producers, distributors, architects, builders, and the public; to provide a uniform basis for guaranteeing compliance through the use of labels or certifications; to avoid delays and misunderstandings; and to effect economies from the producer to the ultimate user through a wider utilization of standard, ponderosa pine doors.

2. In the development of this standard every effort has been made to include designs which will permit freedom of architectural expression. Ponderosa pine doors will continue to be available for all types of architectural designing.

3. To meet the modern trend toward economy and simplification of installation, doors may be specified "Prefit" to the exact size required. (See par 11.) Doors will be mortised for locks and cut for hinges when so specified.

\section{SCOPE}

4. This standard provides minimum specifications for stock ponderosa pine doors in four nominal thicknesses, $3 \frac{1}{4}, 1 \frac{11}{8}, 1 \frac{13}{8}$, and $1 \frac{3}{4}$ inches. It covers construction, grades, and tolerances for these requirements.

4a. There are standard stock lay-outs and designs for the following:

\begin{tabular}{|c|c|c|c|}
\hline Door & Grade & Sizes & Illustrations \\
\hline $\begin{array}{l}\text { Blind or Summer } \\
\text { Combination } \\
\text { Cupboard } \\
\text { Exterior } \\
\text { French or Casement } \\
\text { Flush } \\
\text { Garage } \\
\text { Interior } \\
\text { Sidelights } \\
\text { Storm } \\
\text { Toilet }\end{array}$ & $\begin{array}{l}\text { No. } 1 \\
\text { No. } 1 \\
\text { No. } 1 \\
\text { No. } 1 \text { \& No. } 2 \\
\text { No. } 1 \\
\text { No. } 1 \\
\text { No. } 1 \text { \& Mill Run } \\
\text { No. } 1 \text { \& No. } 2 \\
\text { No. } 1 \\
\text { No. } 1 \text { \& No. } 2 \\
\text { No. } 1\end{array}$ & $\begin{array}{r}\text { Page } \\
7 \\
7 \\
6 \\
6-7 \\
7 \\
7 \\
7 \\
6 \\
6 \\
7 \\
7\end{array}$ & $\begin{array}{r}\text { Page } \\
26 \\
26 \\
23 \\
10-21 \\
20-21 \\
27 \\
24-25 \\
8-9 \\
22 \\
22 \\
25\end{array}$ \\
\hline
\end{tabular}

1 Ponderosa pine, one of the Western pines, has proved over the past 40 years to be highly adaptable for woodwork. This pine is light in color, ranging from creamy-white to straw color. The grain is close, uniform, and resists raising. The surface is even-textured. It takes nails and screws without splitting, is easy to mortise for locks and cut for hinges. It sands to a satin-smooth finish, takes paint, enamel, stain, and varnish, holding them well. The ends and edges do not splinter easily. 


\section{GENERAL REQUIREMENTS}

5. All commercial standard ponderosa pine panel and sash doors shall meet the following requirements: pine.

6. Material.-Doors shall be made of properly kiln-dried ponderosa

7. Workmanship.-Doors shall be well manufactured and machined, with flat faces of stiles, rails, and panels smoothly machine sanded.

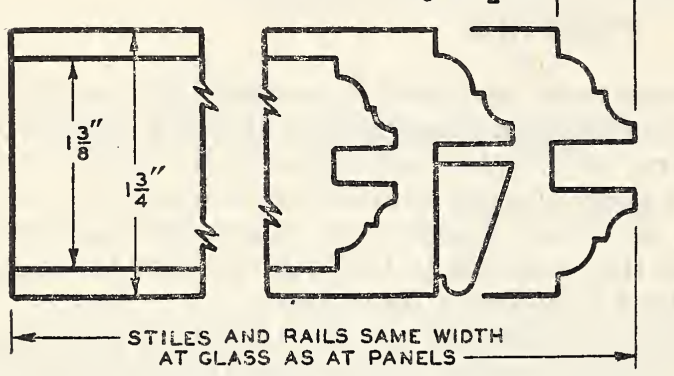

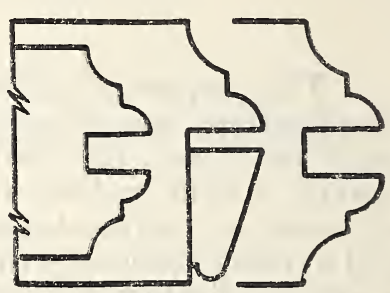

COVE AND BEAD

BEAD AND COVE

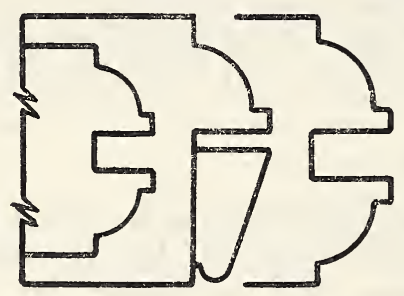

OVOLO "A"

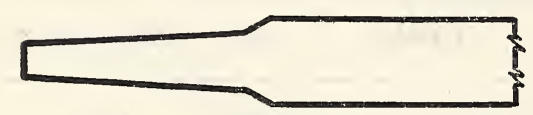

BEVEL RAISED PANEL

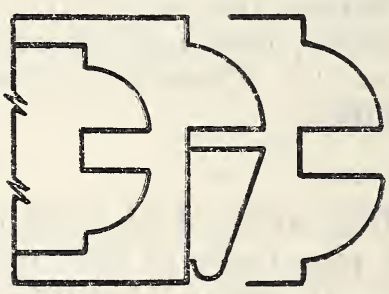

OVOLO "B" OR RULE JOINT

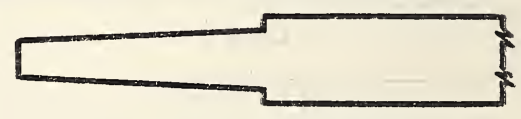

HIP RAISED PANEL

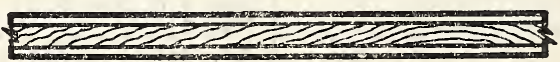

FLAT PLYWOOD PANEL

Frgure 1.-Sticking and panel details.

8. Construction.-Panel and sash doors shall be assembled by what is known as "dowelled construction," that is, stiles and rails to be bored to receive dowels not less than $3 / 8$-inch in diameter by approximately $43 / 4$ inches long for doors $3 / 4$ inch thick, and not less than $1 / 2$ inch in diameter by approximately 5 inches long for doors $1 \frac{1}{8}, 1 \frac{3}{8}$, and $1 \frac{3}{4}$ inches thick. (Except that cupboard doors and narrow stile doors 
may have shorter dowels.) Dowels shall have glued grooves and be to a drive fit. Dowels shall be set in water-resistant glue and shall extend approximately one-half their length into each stile and rail, and assembled under pressure. Because of the fact that all present standard door-boring machines are built for 2\%彳-inch dowel centers, the quantity of dowels used is limited according to the width of the rails and shall be based on the following minimum number of dowels at each end of rails:

Rails under $4 \frac{1}{4}$ inches wide.

Rails $4 \frac{1}{4}$ inches to 7 inches wide

Rails over 7 inches wide.
1 dowel.

2 dowels.

3 dowels, plus one additional dowel for each additional full 3 inches in width.

8a. At the option of the manufacturer, doors may be assembled by what is known as blind-mortised and tenoned construction instead of dowelled construction.

9. Sticking. - Stiles and rails shall have solid sticking. All intersections shall be coped with joints well-fitted. "Cove and Bead," "Bead and Cove," "Ovolo A" or "Ovolo B or Rule Joint" sticking shall be standard on all standard ponderosa pine doors. See figure 1. Imperfect sticking which may develop in machining shall be carefully repaired or neatly replaced. Panels are also illustrated in figure 1.

10. Thicknesses.-Doors shall be of the following thicknesses; and a th ickness tolerance of minus $1 / 16$ inch shall be allowed:

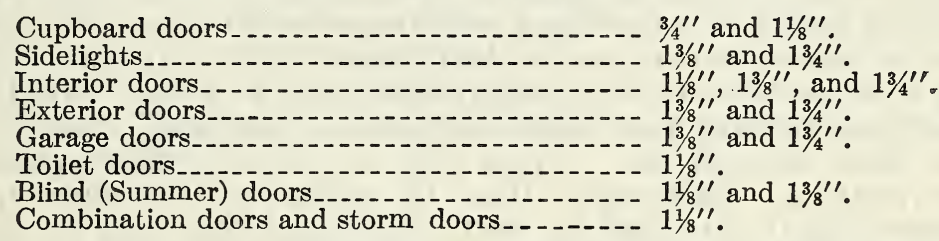

11. Size tolerance. - Unless otherwise specified, a height and width tolerance of plus $1 / 8$ inch shall be allowed. When ordered "Prefit," doors will be made to prefit standard opening widths and heights established by the industry with a tolerance of plus or minus $1 / 32$ inch. "Prefit" doors shall have skid blocks, strips, or other type of protector.

\section{GRADING}

12. All doors shall be graded according to both sides or faces. shipment of any grade shall represent a fair average of that grade.

13. Ordinarily, interior, exterior, and storm doors can be obtained in grades "No. 1" and "No. 2"; cupboard doors, sidelights, casement doors, toilet doors, blind doors, combination doors, and flush doors in grade "No. 1" only; and garage doors in grades "No. 1" and "Mill Run."

GRADE “NO. 1".-Recommended for Natural, Stain, or Paint Finish

14. Stiles and rails.-This stock shall be practically clear. Bright sap, light-brown stain, and light-red kiln burn shall be permitted. Each stile or bottom rail may contain one carefully repaired pitch seam on each side, provided it does not extend through the piece nor 
exceed $2 \frac{1}{2}$ inches in length. Rails wider than $4 \frac{34}{4}$ inches may be glued up with not over one joint up to $93 / 4$ inches, two joints up to 12 inches, and with not more than the same proportion of joints being permitted in wider rails. A water-resistant glue shall be used. Stiles and rails may be solid or veneered at the option of the manufacturer. If veneered, a water-resistant glue shall be used.

15. Panels - flat plywood.-The standard thickness of 3-ply flat plywood panels shall be not less than $1 / 4$ inch after sanding except inner frame and cupboard doors, which shall be not less than $3 / 16$ inch. If ponderosa pine, they shall be "sound and better two sides," according to standard commercial grading rules issued by pine plywood manufacturers; if fir, they shall be "sound two sides," according to Commercial Standard CS45-45; if hardwood, they shall conform to the generally accepted grades of door panels.

16. Panels-solid.- The standard thickness of solid ponderosa pine panels raised two sides shall be not less than $7 / 16$ inch after sanding, and shall conform to the grade of the stiles and rails. Panels wider than $93 / 4$ inches may be glued-up. A water-resistant glue shall be used.

GRADE "NO. 2".- Recommended Primarily for Paint Finish

17. Stiles and rails.-This stock may contain light blue stain, medium-brown stain, or medium-red kiln burn showing on not to exceed 50 percent of the area of any piece, as well as pitch streaks, checks, pitch pockets if carefully slivered, tight sound knots not to exceed $5 / 8$ inch in diameter, and other imperfections, not one of which shall be more serious in nature than those already enumerated. Each stile shall contain one such imperfection, and may have two, but no piece shall contain more than two, and no door shall contain more than eight on each side. Plugs shall be admitted but regarded as imperfections. Rails wider than $4 \frac{3 / 4}{4}$ inches may be glued-up. A water-resistant glue shall be used. Stiles and rails may be solid or veneered at the option of the manufacturer. If veneered, a waterresistant glue shall be used.

18. Panels: flat plywood.-The standard thickness of 3-ply flat plywood panels shall be not less than $1 / 4$ inch after sanding, except inner frame and cupboard doors, which shall be not less than $3 / 16$ inch. If ponderosa pine, they shall be "sound and better two sides" according to standard commercial grading rules issued by pine plywood manufacturers; if fir, they shall be "sound two sides," according to Commercial Standard CS45-45.

19. Panels: solid.-The standard thickness of solid ponderosa pine panels raised two sides shall be not less than $7 / 16$ inch after sanding and shall conform to the grade of the stiles and rails. Panels wider than $93 / 4$ inches may be glued-up. A water-resistant glue shall be used. 
GRADE "MILL RUN".--Recommended for Paint Finish Only (Garage Doors Only)

20. "Mill Run" grade may contain blue stain, brown stain or redkiln burn, worm holes, checks, pitch streaks, pitch pockets, fine shake, tight sound knots not to exceed 2 inches in diameter, and other imperfections, none of which shall be more serious in nature than those already enumerated.

21. Panels: flat plywood.-The standard thickness of 3-ply flat plywood panels shall be not less than $1 / 4$ inch after sanding. If ponderosa pine, they shall be "sound and better two sides" according to standard commercial grading rules issued by pine plywood manufacturers; if fir, they shall be "sound two sides": according to Commercial Standard CS45-45.

22. Panels: solid.-The standard thickness of solid ponderosa pine panels raised two sides shall be not less than $7 / 16$ inch after sanding and shall conform to the grade of the stiles and rails. Panels wider than $9 \frac{3}{4}$ inches may be glued-up. A water-resistant glue shall be used.

\section{DESIGNS AND LAY-OUTS}

23. Measurements for stiles, rails, mullions, and muntins shown in lay-outs are over-all (face measurement plus the sticking). A tolerance of $1 / 8$ inch in width shall be permitted. Unless otherwise specified, glass measurements may vary not more than $1 / 4$ inch from those shown in the lay-outs. (These tolerances allow for variations in different manufacturers' practices.)

24. Interior doors of any design narrower than 1 foot 6 inches in width will be furnished with stiles $3 \frac{3}{4}$ inches over-all width, unless otherwise specified. 
TABLE 1.-Standard sizes

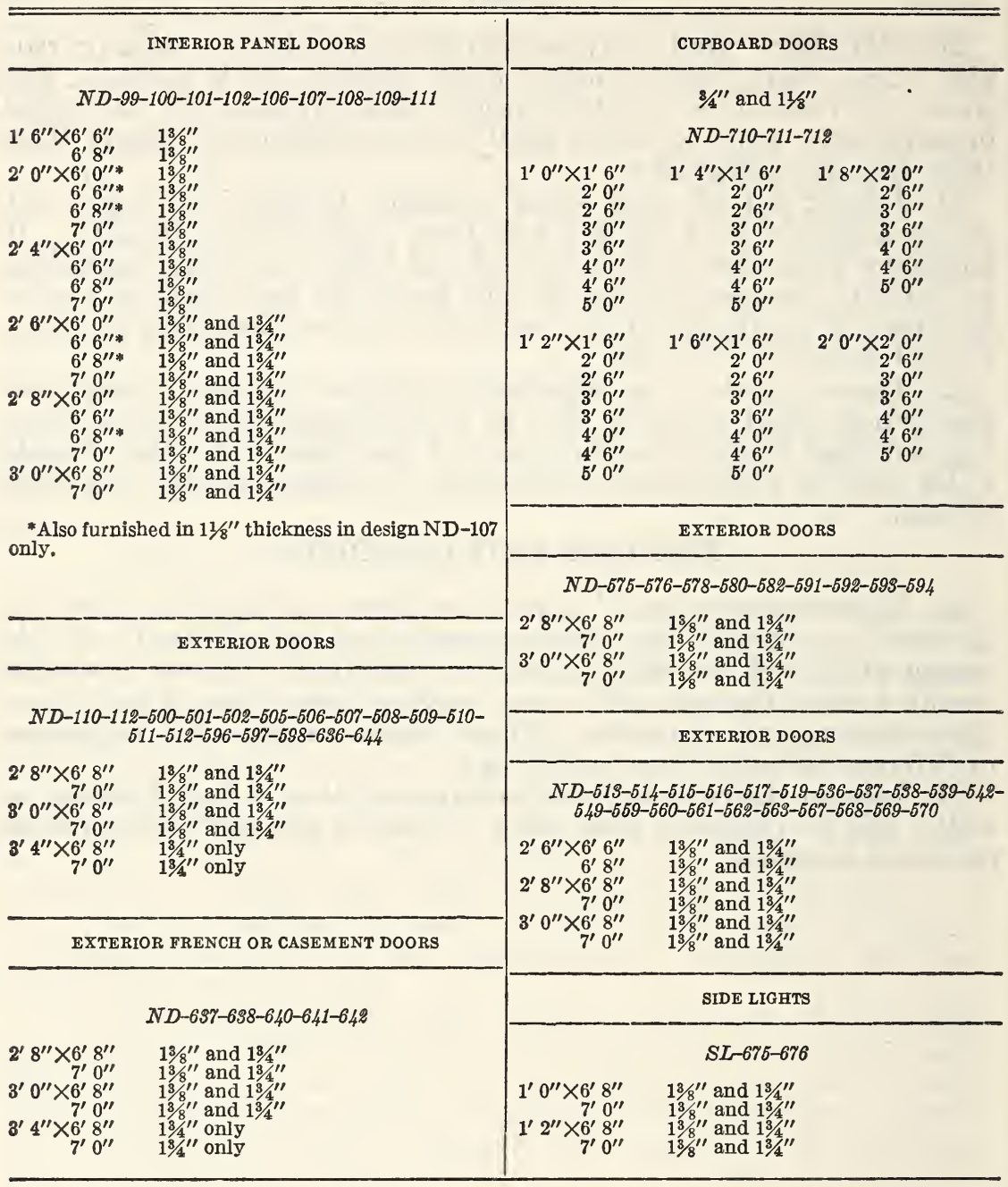


TABLE 1.-Standard sizes-Continued

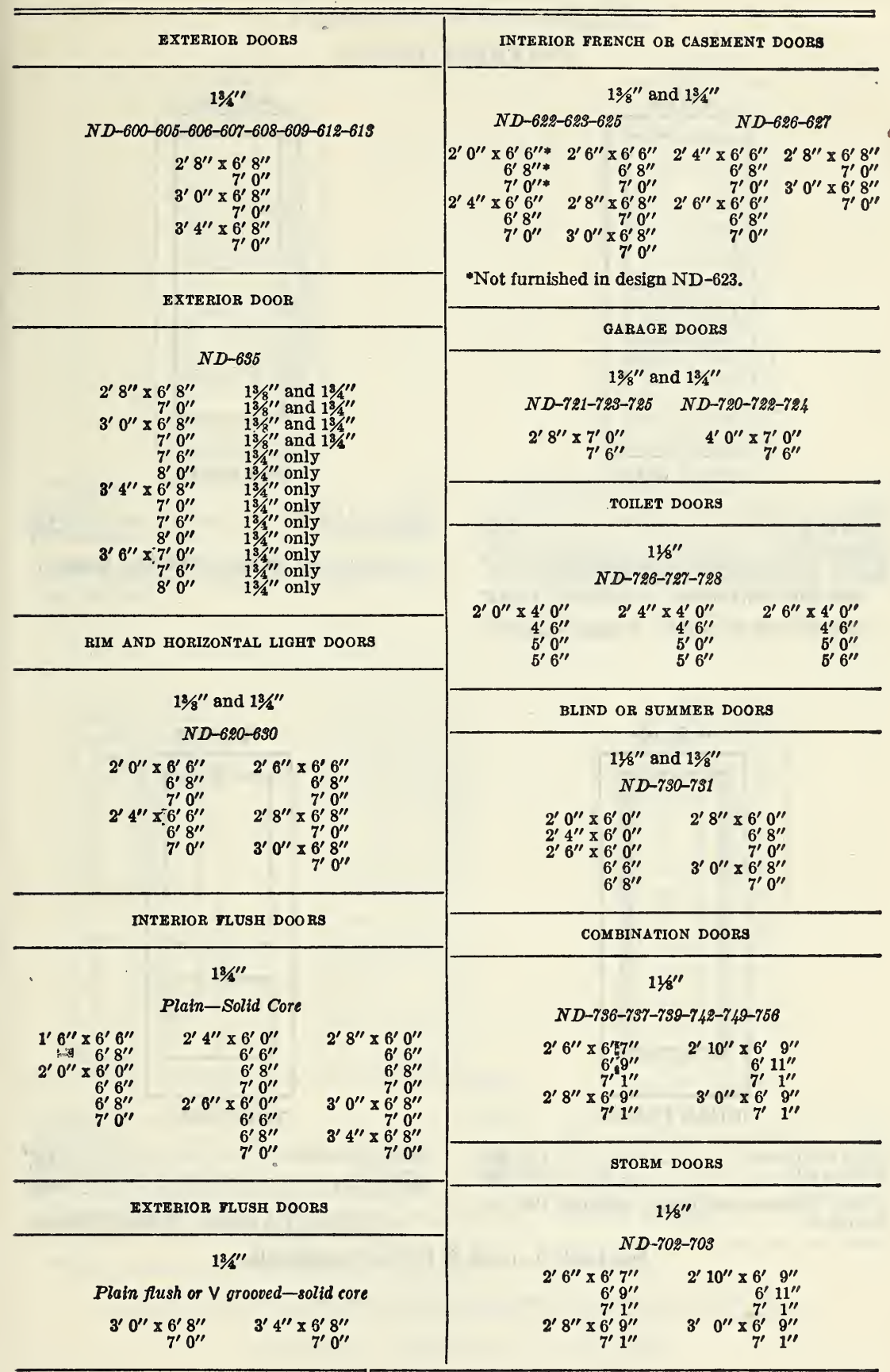




\section{INTERIOR DOORS}

\section{N.D. 99}

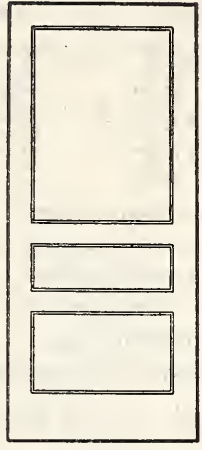

THREE PANEL

Stiles and top rail Cross rails

Bottom rail.

Height of center panel, including sticking. $815 / 16^{\prime \prime}$ Height from bottom of door to top of

upper cross rail, sticking not included.- $4111 / 16^{\prime \prime}$

3-ply plywood flat panels. Sticking: Standard.

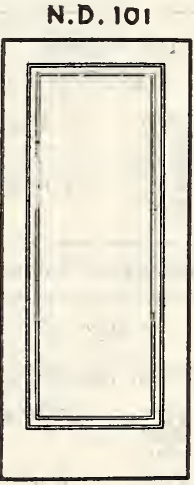

INNER FRAME

Stiles and top rail $434^{\prime \prime}$ face Bottom rail $91 / 4^{\prime \prime}$ or $91 / 2^{\prime \prime}$ face 3-ply plywood flat panel. Sticking: P\&G or Standard.
N.D. 100

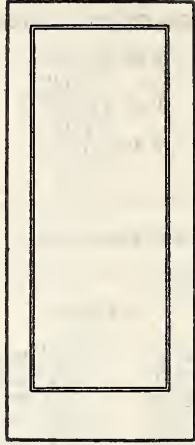

ONE PANEL

Stiles and top rail Bottom rail
N.D. 102

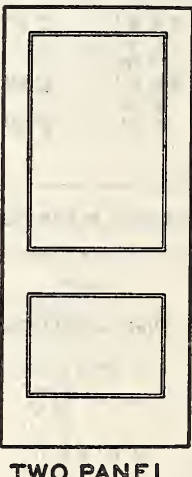

TWO PANEL

Stiles and top rail

Lock rail

Bottom rail

3-ply plywood flat panels. Sticking:-Standard.

See table_1, page 6, for sizes available. 


\section{INTERIOR DOORS-Continued}

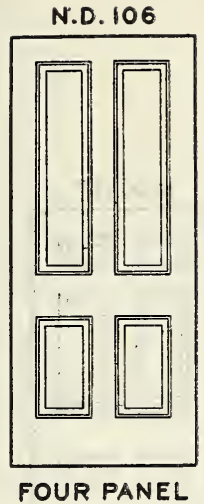

Stiles and top rail

Lock rail.

Muntins

Bottom rail

Raised panels 2 sides. Can also be furnished with 3-ply plywood flat panels, if desired. Sticking: Standard.

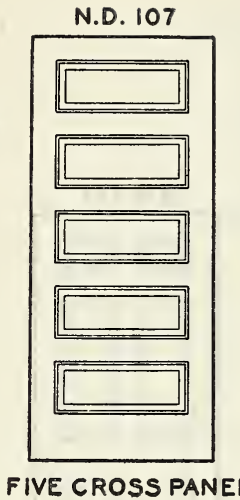

Stiles and top rail

$43 / 4$

Bottom rail ...................... $958^{\prime \prime}$

Raised panels 2 sides. Can also be furnished with 3-ply plywood flat panels, if desired. Stick ing: Standard.

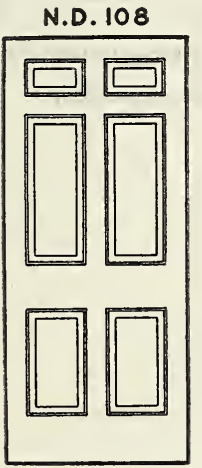

\section{SIXPANEL COLONIAL EICHT PANEL COLONIAL}

Stiles and top rail

Lock rail

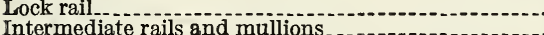

Bottom rail

N.D. III

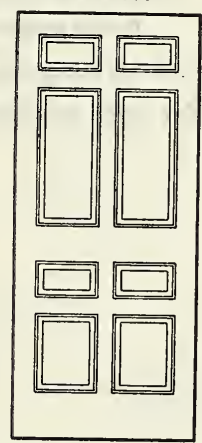

Height of top panels over-all ......................... 71/8"

Raised panels 2 sides. Can also be furnished with 3-ply plywood flat panels. Sticking: Standard.

Doors $1^{\prime} 6^{\prime \prime}$ and narrower are made 1 panel wide.

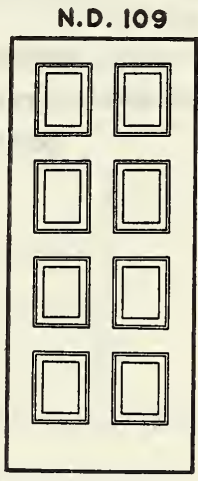

EIGHT PANEL

Stiles and top rail ....... $434^{\prime \prime}$

Intermediate rails and mulions..... $37 / 8^{\prime \prime}$ Bottom rail............ 95/8

Raised panels 2 sides. Can also be furnished with 3-ply plywood flat panels. Sticking: Standard.

Doors $1^{\prime} 6^{\prime \prime}$ and narrower are made 1 panel wide.

$45 / 8^{\prime \prime}$ intermediate rails and mullions are optional with some manufacturers.

Bottom and lock rails for N. D. 108 and N. D. 111 can be reversed when so specified.

See table 1 , page 6 , for sizes available. 


\section{EXTERIOR DOORS}

N.D. 110

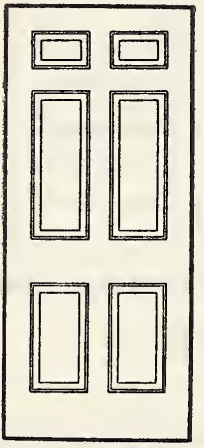

SIX PANEL COLONIAL

Stiles and top rail

Lock rail .

Intermediater

Bottom rail

Panel thickness.

of top panels over-al

Raised panels 2 sides. Sticking: Standard.
N.D. 112

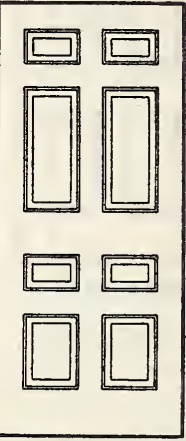

EIGHT PANEL COLONIAL

Stiles and top rail

Lock rail top rail

Intermediate rails and mullions

Bottom rail Height of small panels

Raised panels 2 sides. Sticking: Standard

Bottom and lock rails for N. D. 110 and N. D. 112 can be reversed when so specified.

See table 1, page 5, for sizes available. 


\section{EXTERIOR DOORS-Continued}

N.D. 500

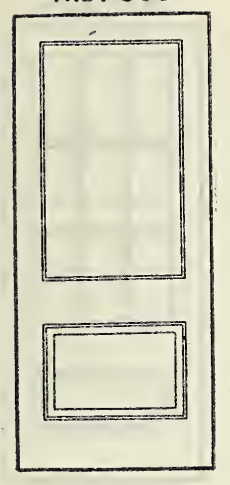

N.D. 501

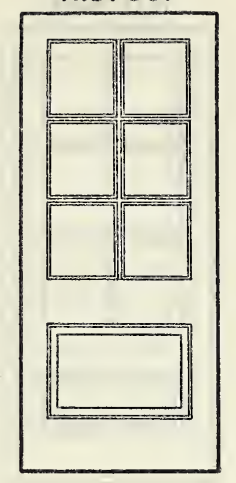

N.D. 502

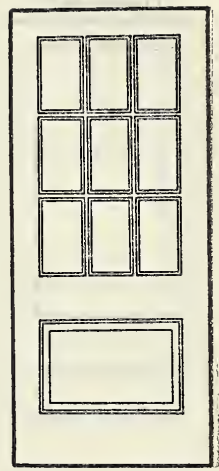

N.D. 505

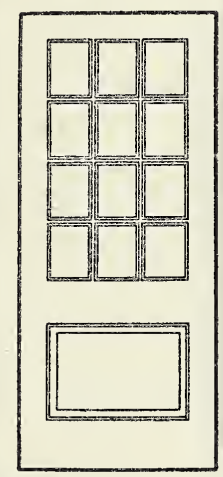

Stiles and top rail Lock rail

Bottom rail.
N.D. 506

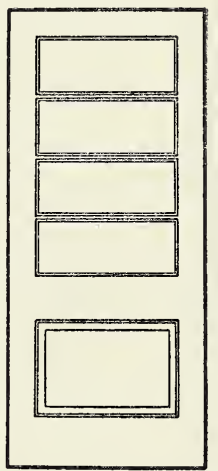

$51 / 2^{\prime \prime}$

Raised panel 2 sides. Can also be furnished with 3-ply plywood flat panel, if desired. Sticking: Standard

Door No.

N. D. 500

N. D. 501

N. D. 502

N. D. 505

N. D. 506

\section{Approximate glass size}

Size of door $3^{\prime} 0^{\prime \prime} \times 6^{\prime} 8^{\prime \prime}$

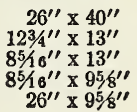

$26^{\prime \prime} \times 958^{\prime \prime}$
Size of door $3^{\prime} 0^{\prime \prime} \times 7^{\prime} 0^{\prime \prime}$

$$
\begin{aligned}
& \begin{array}{r}
22^{\prime \prime} \times 40^{\prime \prime} \\
10^{3 / \prime} 4^{\prime \prime} \times 13^{\prime \prime}
\end{array} \\
& 7^{\prime \prime} \times 13^{\prime \prime} \\
& \begin{array}{r}
7 \prime \prime \times 18^{\prime \prime} \\
22^{\prime \prime} \times 958^{\prime \prime}
\end{array}
\end{aligned}
$$

Beads for glass included.

$8^{\prime \prime}$ lock rail can be furnished when so specified.

See table 1, page 6 , for sizes available. 


\section{EXTERIOR DOORS-Continued}

N.D. 507

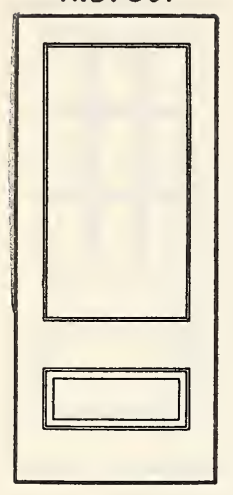

N.D. 510

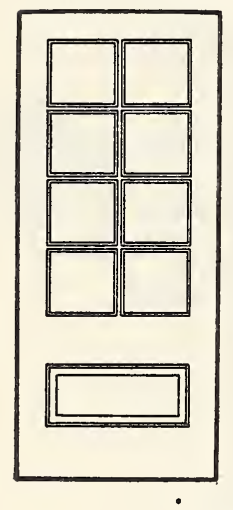

N.D. 508

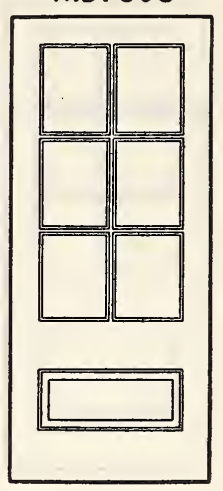

N.D. 5 II

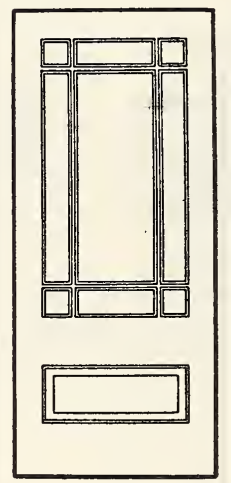

N.D. 509

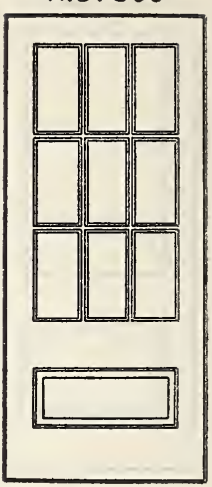

N.D. 512

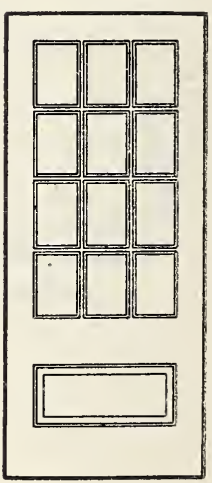

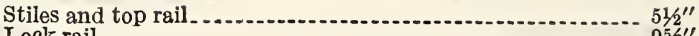

Lock rail .

Bottom rail...

Raised panel 2 sides. Can also be furnished with 3-ply plywood flat panel, if desired. Sticking: Standard.

Door No.

N. D. 507

N. D. 508

N. D. 509

N. D. 510

N. D. 511

N. D. 512

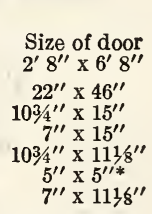

Approximate glass size Size of door $3^{\prime} 0^{\prime \prime} \times 6^{\prime} 8^{\prime \prime}$ $26^{\prime \prime} \times 46^{\prime \prime}$ $123 / 4^{\prime \prime} \times 15^{\prime \prime}$ $8516^{\prime \prime} \times 15^{\prime \prime}$ $1234^{\prime \prime} \times 1118^{\prime \prime}$ $5^{\prime \prime} \times 5^{\prime \prime *}$ 85 16" $^{\prime \prime} \times 1118^{\prime \prime}$

Size of door $3^{\prime} 0^{\prime \prime} \times 7^{\prime} 0^{\prime \prime}$ $26^{\prime \prime} \times 50^{\prime \prime}$ $1234^{\prime \prime} \times 165 / 16^{\prime \prime}$ $856^{\prime \prime} \times 1656^{\prime \prime}$ $123 / 4^{\prime \prime} \times 1218^{\prime \prime}$ $5^{\prime \prime} \times 5^{\prime \prime *}$ $85 / 16^{\prime \prime} \times 1218^{\prime \prime}$

Beads for glass included. * Corner lights.

' 8 " lock rail can be furnished when so specified.

See table 1 , page 6 , for sizes available. 


\section{EXTERIOR DOORS-Continued}

N.D. 513

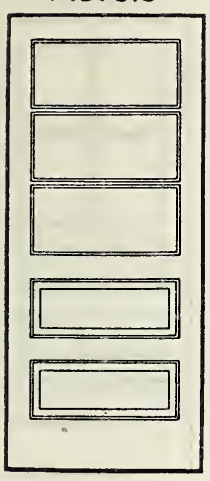

N.D. 516

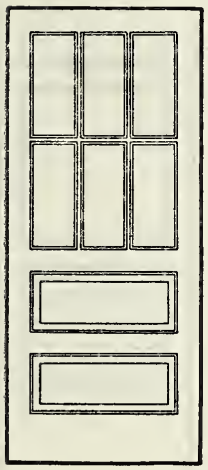

N.D. 514

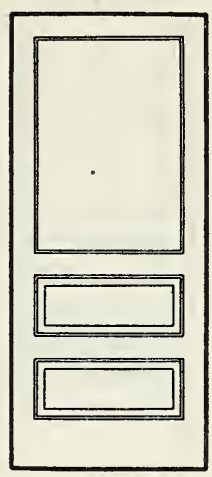

N.D. 517

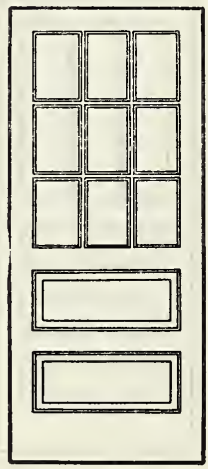

N.D. 515

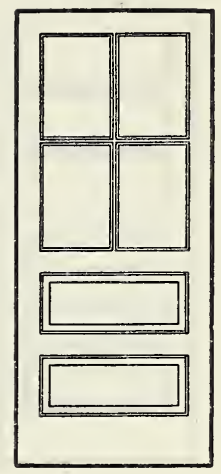

N.D. 519

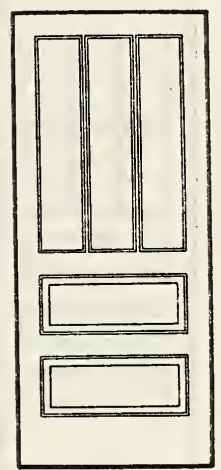

Stiles and top rail_

Lock rail

$45 / 8^{\prime \prime}$

Bottom rail

Raised panels 2 sides. Can also be furnished with 3-ply plywood flat panels, desired. Sticking: Standard.

\section{Door No.}

N. D. 513

N. D. 514

N. D. 515

N. D. 516

N. D. 517

N. D. 519

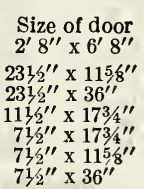

Approximate glass size

$\begin{array}{cc}\text { Size of door } & \text { Size of door } \\ 3^{\prime} 0^{\prime \prime} \times 6^{\prime} 8^{\prime \prime} & 3^{\prime} 0^{\prime \prime} \times 7^{\prime} 0^{\prime \prime} \\ 2712^{\prime \prime} \times 1158^{\prime \prime} & 2712^{\prime \prime} \times 13^{\prime \prime} \\ 2712^{\prime \prime} \times 36^{\prime \prime} & 2712^{\prime \prime} \times 40^{\prime \prime} \\ 1312^{\prime \prime} \times 1734^{\prime \prime} & 1312^{\prime \prime} \times 1934^{\prime \prime} \\ 81316^{\prime \prime} \times 173,4^{\prime \prime} & 81316^{\prime \prime} \times 1934^{\prime \prime} \\ 81316^{\prime \prime} \times 11558^{\prime \prime} & 81316^{\prime \prime} \times 13^{\prime \prime} \\ 813 / 16^{\prime \prime} \times 36^{\prime \prime} & 813 / 16^{\prime \prime} \times 40^{\prime \prime}\end{array}$

Beads for glass included.

See table 1, page 6 , for sizes available. 
EXTERIOR DOORS-Continued

N.D. 536

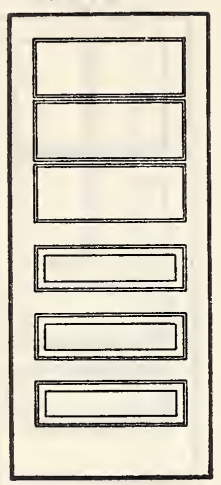

N.D. 539

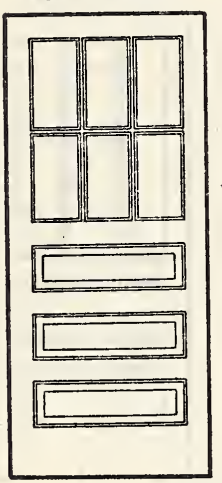

N.D. 537

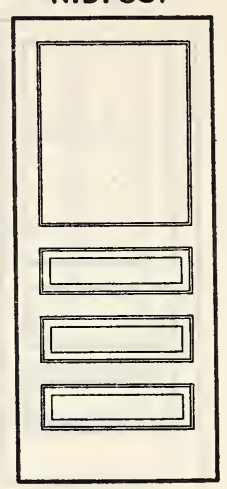

N.D. 542

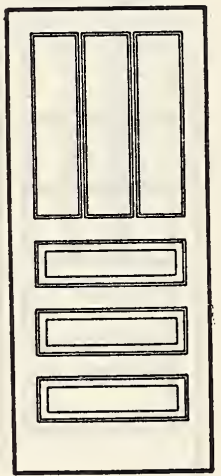

N.D. 538

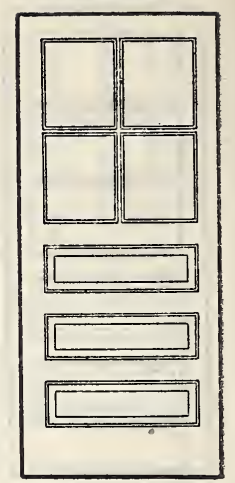

N.D. 549

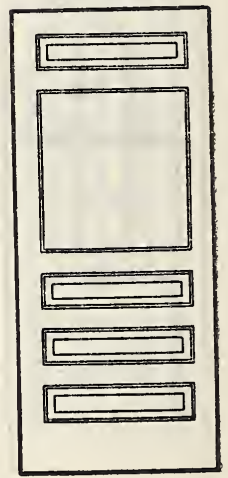

Stiles and top rail.

Cross rails

to ren

$434^{\prime \prime}$

Raised panels 2 sides. Can also be furnished with 3-ply plywood flat panels, if desired. Sticking: Standard.

\section{Door No.}

N. D. 536

N. D. 537

N. D. 539

N. D. 542

N. D. 549

Size of door
$2^{\prime} 6^{\prime \prime} \times 6^{\prime} 6^{\prime \prime}$
$211^{\prime \prime \prime} \times 9^{\prime \prime}$
$211^{\prime \prime} \times 28^{\prime \prime}$
$101 / 2^{\prime \prime} \times 1334^{\prime \prime}$
$613 / 16^{\prime \prime} \times 1334^{\prime \prime}$
$613 / 6^{\prime \prime} \times 28^{\prime \prime}$
$2112^{\prime \prime} \times 24^{\prime \prime}$

A pproximate glass size

\begin{tabular}{|c|c|}
\hline $\begin{array}{l}\text { Size of door } \\
2^{\prime} 8^{\prime \prime} \times 6^{\prime} 8^{\prime \prime}\end{array}$ & $\begin{array}{l}\text { Size of door } \\
3^{\prime} 0^{\prime \prime} \times 6^{\prime} 8^{\prime \prime}\end{array}$ \\
\hline 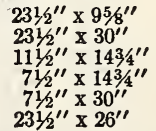 & 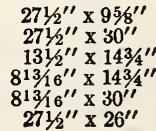 \\
\hline
\end{tabular}

$2312^{\prime \prime} \times 26^{\prime \prime}$
Size of door $3^{\prime} 0^{\prime \prime} \times 7^{\prime} 0^{\prime \prime}$ $2712^{\prime \prime} \times 11^{\prime \prime}$ $2712^{\prime \prime} \times 34^{\prime \prime}$ $1312^{\prime \prime} \times 163 / 4^{\prime \prime}$ $813 / 16^{\prime \prime} \times 1634^{\prime \prime}$ $813 / 16^{\prime \prime} \times 34^{\prime \prime}$ $2712^{\prime \prime} \times 30^{\prime \prime}$

Beads for glass included

See table 1, page 6, for sizes available. 
EXTERIOR DOORS-Continued

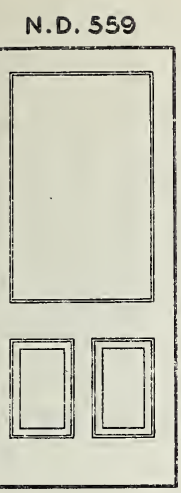

N.D. 560

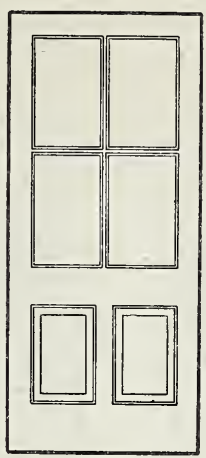

N.D. 561

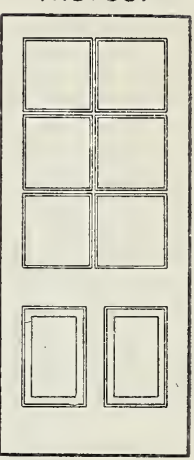

N.D. 562

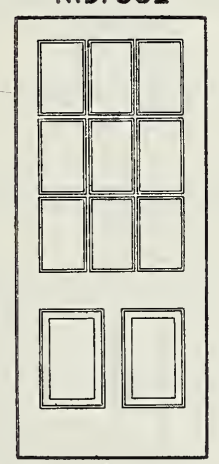

Stiles and top rail Lock rail

Muntins.

Bottom rail
N.D. 563

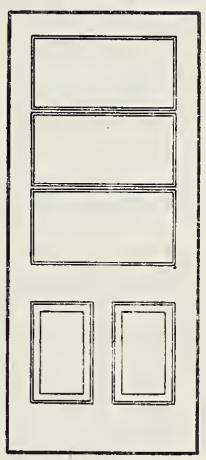

Raised panels 2 sides. Can also be furnished with 3-ply plywood flat panels, if desired. Sticking: Standard.

Door No.

N. D. 559

N. D. 560

N. D. 561

N. D. 562

Size of door
$2^{\prime} 6^{\prime \prime} \times 6^{\prime} 6^{\prime \prime}$
$211 / 2^{\prime \prime} \times 38^{\prime \prime}$
$1012^{\prime \prime} \times 18344^{\prime \prime}$
$101 / 2^{\prime \prime} \times 125 / 16^{\prime \prime}$
$613 / 16^{\prime \prime} \times 12516^{\prime \prime}$
$211 / 2^{\prime \prime} \times 1256^{\prime \prime}$

Beads for glass included
Size of door $2712^{\prime \prime} \times 44^{\prime \prime}$ $131 / 2^{\prime \prime} \times 2134^{\prime \prime}$ $1312^{\prime \prime} \times 14516^{\prime \prime}$ $813 / 16^{\prime \prime} \times 145 / 16^{\prime \prime}$ $2712^{\prime \prime} \times 14516^{\prime \prime}$

See table 1 , page 6 , for sizes available. 


\section{EXTERIOR DOORS-Continued}
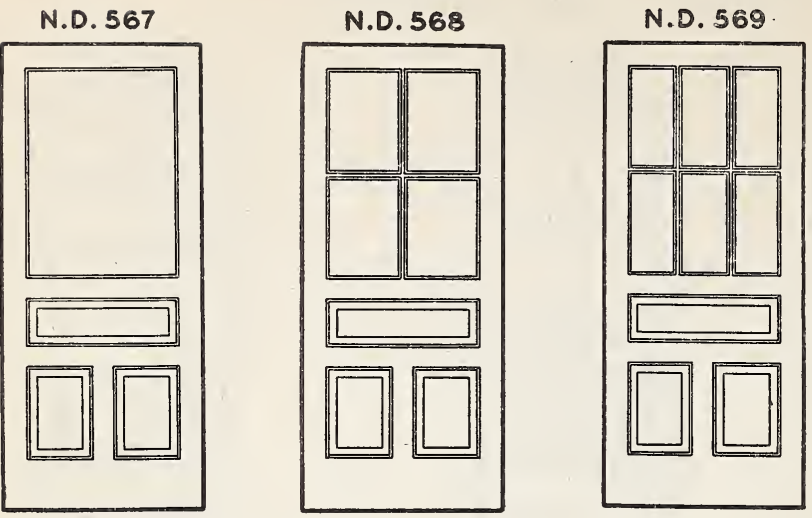

N.D. 570

Stiles and top rail.

Cross rails.

Muntins

(1)

Bottom rail

Raised panels 2 sides. Can also be furnished with 3-ply plywood flat panels, if desired. Sticking: Standard.

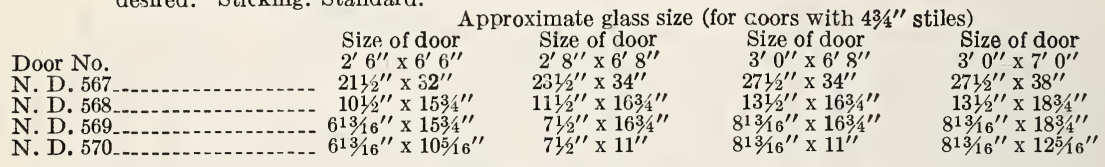

Beads for glass included.

Above also supplied with $51 / 2^{\prime \prime}$ stiles and top rail, $53 / 8^{\prime \prime}$ cross rails and muntins when so specified. Top panel made 2 panels wide, when so specified.
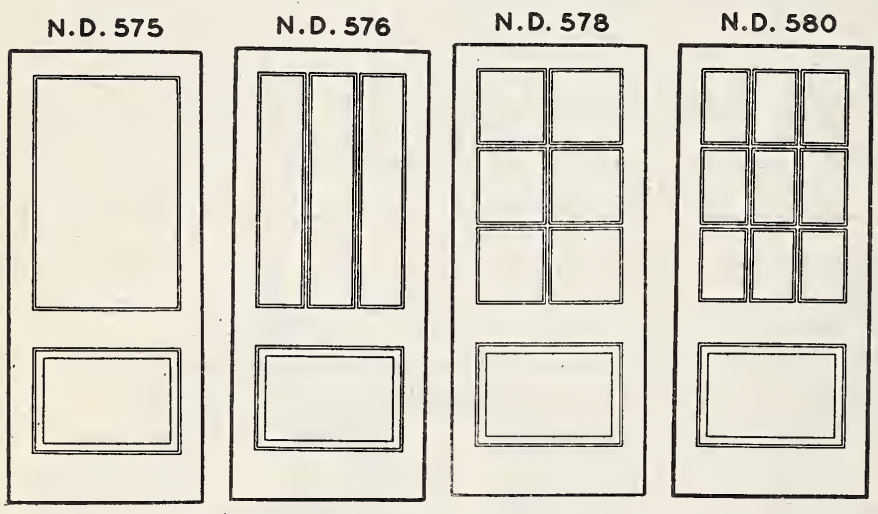

N.D. 582

Stiles and top rail

Lock rail

Raised panel 2 sides. Can also be furnished with 3-ply plywood flat panels, if desired. Sticking: Standard

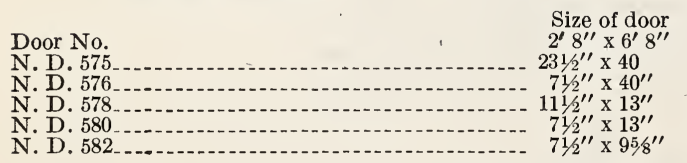

$$
\begin{array}{cc}
\text { Approximate glass size } & \\
\text { Size of door } & \text { Size of door } \\
3^{\prime} 0^{\prime \prime} \times 6^{\prime} 8^{\prime \prime} & 3^{\prime} 0^{\prime \prime} \times 7^{\prime} 0^{\prime \prime} \\
2712^{\prime \prime} \times 40^{\prime \prime} & 2712^{\prime \prime} \times 44^{\prime \prime} \\
813 / 1^{\prime \prime} \times 10^{\prime \prime} & 813 / 16^{\prime \prime} \times 44^{\prime \prime} \\
131 / 2^{\prime \prime} \times 13^{\prime \prime} & 1312^{\prime \prime} \times 146^{\prime \prime} \\
813 / 6^{\prime \prime} \times 13^{\prime \prime} & 813 / 16^{\prime \prime} \times 146^{\prime \prime} \times 16^{\prime \prime} \\
813 / 16^{\prime \prime} \times 95 / 8^{\prime \prime} & 813 / 16^{\prime \prime} \times 10 \% 8^{\prime \prime}
\end{array}
$$

Beads for glass included.

See table 1, page 6, for sizes available. 


\section{EXTERIOR DOORS-Continued}
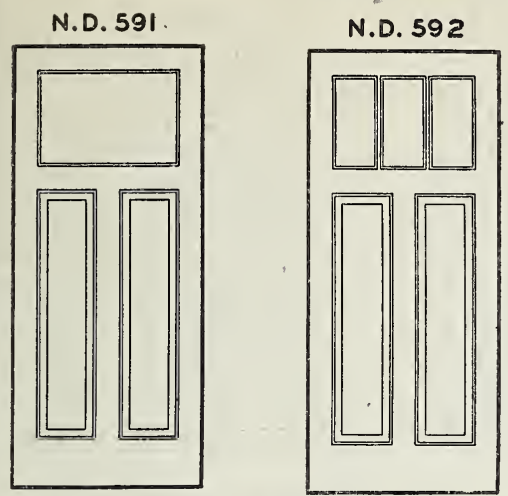

N.D. 593

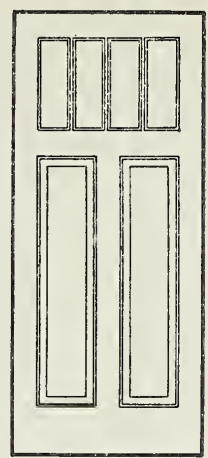

N.D. 594
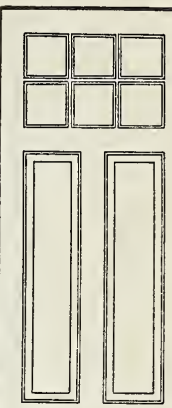

Stiles and top rail

Mullion and cross rail

Bottom rail

Raised panels 2 sides. Can also be furnished with 3-ply plywood flat panels, if desired Sticking: Standard.

Door No.

N. D. 591

N. D. 592

N. D. 593

N. D. 594
Approximate glass size (for doors with $51 / 2 "$ stiles) $\begin{array}{ll}\text { Size of door } & \text { Size of door } \\ 2^{\prime} 8^{\prime \prime} \times 6^{\prime} 8^{\prime \prime} & 3^{\prime} 0^{\prime \prime} \times 6^{\prime} 8^{\prime \prime}\end{array}$ $\begin{aligned} 22^{\prime \prime} & \times 18^{\prime \prime} \\ 7^{\prime \prime} & \times 18^{\prime \prime}\end{aligned}$

$\begin{array}{ll}8^{\prime \prime} & \times 18^{\prime \prime} \\ 7^{\prime \prime} & \times 84^{\prime \prime}\end{array}$ $26^{\prime \prime} \times 18^{\prime \prime}$

$85 / 16^{\prime \prime} \times 18^{\prime \prime}$

$61 / 8^{\prime \prime} \times 18^{\prime \prime}$ $856^{\prime \prime} \times 834^{\prime \prime}$

\begin{abstract}
Size of doo $3^{\prime} 0^{\prime \prime} \times 7^{\prime} 0^{\prime \prime}$ $26^{\prime \prime} \times 18^{\prime \prime}$ $85 / 16^{\prime \prime} \times 18^{\prime \prime}$ $61 / 8^{\prime \prime} \times 18^{\prime \prime}$ $85 / 16 " \times 834^{\prime \prime}$
\end{abstract}

Beads for glass included.

Above also supplied with $434^{\prime \prime}$ stiles and top rail, $4 \frac{5}{3^{\prime \prime}}$ mullion and cross rail, when so specified.

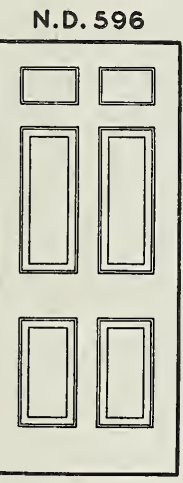

BAR. $5-3 / 8^{\prime \prime}$
N.D. 597

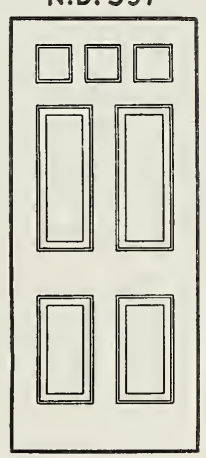

BARS
N.D. 598

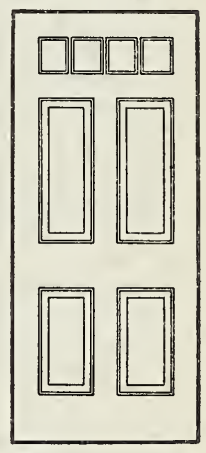

BARS.

Stiles and top rail

Lock rail

Top cross rail and mullions

Bottom rail

3/4" Raised panels 2 sides. Sticking: Standard.

Door No.

N. D. 596

N. D. 597

N. D. 598
Size of door
$2^{\prime} 8^{\prime \prime} \times 6^{\prime} 8^{\prime \prime}$

$834^{\prime \prime} \times 71 / 8^{\prime}$

$534^{\prime \prime} \times 718^{\prime \prime}$

$51 / 8^{\prime \prime} \times 718^{\prime \prime}$

Approximate glass size

$$
\begin{array}{rr}
\text { Size of door } & \text { Size of door } \\
3^{\prime} 0^{\prime \prime} \times 6^{\prime} 8^{\prime \prime} & 3^{\prime} 0^{\prime \prime} \times 7^{\prime} 0^{\prime \prime} \\
1034^{\prime \prime} \times 71 / 8^{\prime \prime} & 1034^{\prime \prime} \times 71 / 8^{\prime \prime} \\
7^{\prime \prime} \times 71 / 8^{\prime \prime} & 7^{\prime \prime} \times 71 / 8^{\prime \prime} \\
61 / 8^{\prime} \times 71 / 8^{\prime \prime} & 61 / 8^{\prime \prime} \times 71 / 8^{\prime \prime}
\end{array}
$$

Beads for glass included.

Bottom and lock rails can be reversed when so specified.

See table 1, page 6 , for sizes available. 


\section{EXTERIOR DOORS-Continued}

N.D. 600

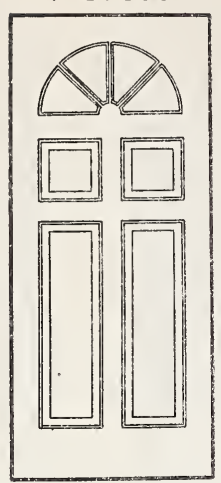

Stiles and top

Top cross rall

Bottom rail.

Raised panels 2 sides. Can also be furnished with 3-ply plywood flat panels, if desired. Sticklng: Standard:

Size of door

$2^{\prime} 8^{\prime \prime} \times 6^{\prime} 8^{\prime \prime}$

$3_{1} 0^{\prime \prime} \times 6^{\prime} 8^{\prime \prime}$

$3^{\prime} 0^{\prime \prime} \times 7^{\prime} 0^{\prime \prime}$

Approx. glass opg. $22^{\prime \prime} \times 11^{\prime \prime}$ $26^{\prime \prime} \times 13^{\prime \prime}$

Beads for glass included.
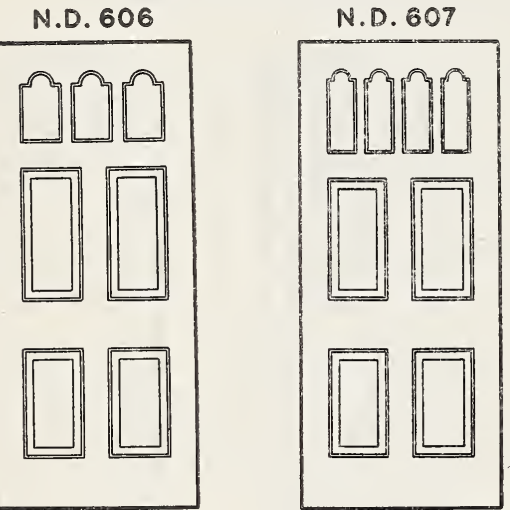

Stiles and top rail _......... $51 / 2^{\prime \prime}$ Lock rail Top cross rail and mullions _......... Bottom rail

$3 / 4^{\prime \prime}$ raised panels 2 sides. Sticking: Standard. Approx. glass size

$\begin{array}{lrr}\text { Size of door } & \text { N. D. } 606 & \text { N. D. } 607 \\ 2^{\prime} 8^{\prime \prime} \times 6^{\prime} 8^{\prime \prime} & 63 / 16^{\prime \prime} \times 9^{\prime \prime} & 43 / 8^{\prime \prime} \times 11^{\prime \prime} \\ 3^{\prime} 0^{\prime \prime} \times 6^{\prime} 8^{\prime \prime} \ldots \ldots \ldots \ldots & 71 / 2^{\prime \prime} \times 9^{\prime \prime} & 53 / 8^{\prime \prime} \times 11^{\prime \prime} \\ 3^{\prime} 0^{\prime \prime} \times 7^{\prime} 0^{\prime \prime} \ldots \ldots \ldots \ldots . & 71 / 2^{\prime \prime} \times 13^{\prime \prime} & 53 / 8^{\prime \prime} \times 15^{\prime \prime}\end{array}$

Beads for glass included.

Bottom and lock rails can be reversed when so specified.
N.D. 605

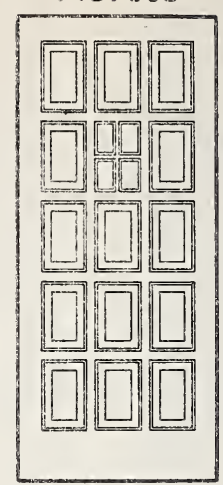

Stiles and top rail _. $43 / 4^{\prime \prime}$ Cross rails and muntins Bottom rail

Raised panels 2 sides. Can also be furnished with 3-ply plywood flat panels, if desired. Sticking: Standard.

Size of door $2^{\prime} 8^{\prime \prime} \times 6^{\prime} 8^{\prime \prime}$ $3^{\prime} 0^{\prime \prime} \times 6^{\prime} 8^{\prime \prime}$ Approx. glass opg. $3^{\prime} 0^{\prime \prime} \times 7^{\prime} 0^{\prime \prime}$ $63 / 4^{\prime \prime} \times 117 / 8^{\prime \prime}$

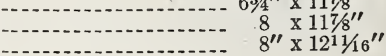

Beads for glass included.

N.D. 608

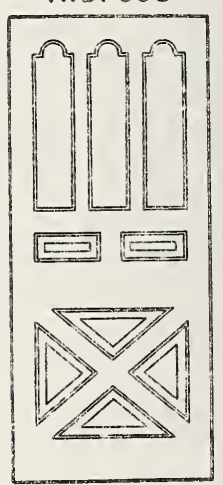

Stiles and top rail ..................... $43 / 4^{\prime \prime}$

Lock rail.

Cross rail and mullions................ $458^{\prime \prime}$

Bottom rail . . . .

Bars..................

$11 / 8^{\prime \prime}$ heavy raised panels 2 sides. Sticking: Standard.

Size of door $2^{\prime} 8^{\prime \prime} \times 6,8^{\prime \prime} \ldots \ldots 1 / 8^{\prime \prime} \times 267 / 8^{\prime \prime}$

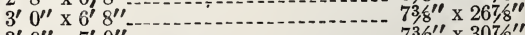
$3^{\prime} 0^{\prime \prime} \times 7^{\prime} 0^{\prime \prime}-\ldots$

Beads for glass included.

Also supplied ${ }^{\mathrm{S}}$ with $3 / 4^{\prime \prime}$ raised panels when sa specified.

See table 1, page 7, for sizes available. 


\section{EXTERIOR DOORS-Continued}

\section{N.D. 609}

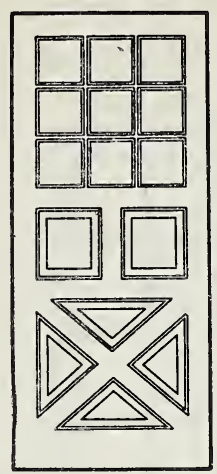

St1les and top rail Lock rail . .

Cross rail and mullions........................ $458^{\prime \prime}$

Bottom rail ..........

$13 / 8^{\prime \prime}$ hip raised panels 2 sides. Sticking: Standard.

Size of door

$2^{\prime} 8{ }^{\prime} \times 6^{\prime} 8^{\prime \prime}$

$3^{\prime} 0^{\prime \prime} \times 6^{\prime} 8^{\prime \prime}$

Approx. glass size $712^{\prime \prime} \times 758^{\prime \prime}$
$813 / 16^{\prime \prime} \times 758^{\prime \prime}$ $813111 \times 9^{\prime \prime}$

Beads for glass included.

Also supplied with $134^{\prime \prime}$ raised panels, when so specified.

\section{N.D. 612}

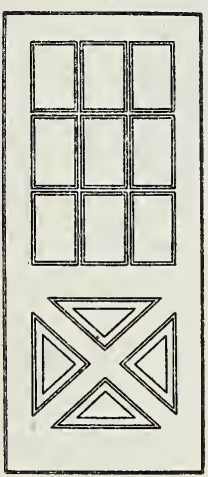

N.D. 613

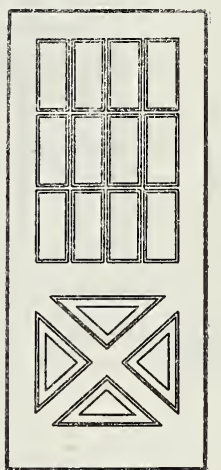

Stiles and top rail

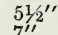

Lock rail.

Mullions

Bottom rail

$118^{\prime \prime}$ heavy raised panels 2 sides. Sticking: Standard.

$$
\begin{aligned}
& \text { Approx. glass size } \\
& \begin{array}{lll}
\text { Size of door } & \text { N. D. } 612 & \text { N. D. } 613
\end{array} \\
& 2^{\prime} 8^{\prime \prime} \times 6^{\prime} 8^{\prime \prime}-\ldots . . .-7^{\prime \prime} \times 12^{\prime \prime} \quad 51 / 8^{\prime \prime} \times 12^{\prime \prime} \\
& 3^{\prime} 0^{\prime \prime} \times 6^{\prime} 8^{\prime}-\ldots . . .-. . .85 / 16^{\prime} \times 12^{\prime \prime} \quad 61 / 8^{\prime \prime}{ }_{6}^{\prime} 12^{\prime \prime} \\
& 3^{\prime} 0^{\prime \prime} \times 7^{\prime} 0^{\prime \prime} \ldots . . . . .-85 / 16^{\prime \prime} \times 135 / 16^{\prime \prime} \quad 618^{\prime \prime}{ }_{4} \times 135 / 16^{\prime \prime}
\end{aligned}
$$

Beads for glass included.

Also supplied with $34^{\prime \prime}$ raised panels, when so specified.

See table 1, page 7, for sizes available. 


\section{EXTERIOR DOORS-Continued}

(Also for Interior)

Rim, horizontal light, French, or casement

N.D. 620

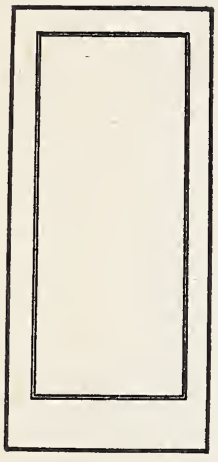

N.D. 622

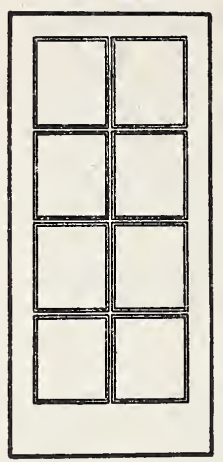

N.D. 623

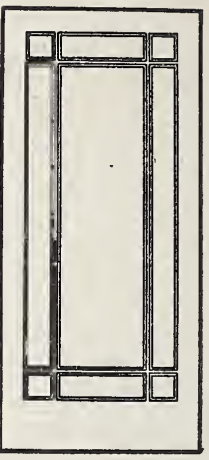

N.D. 630

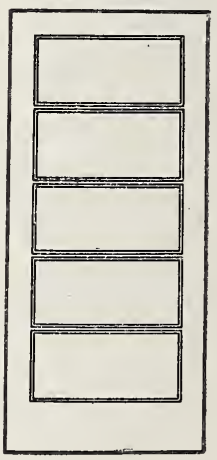

Stiles and top rail

N.D. 627

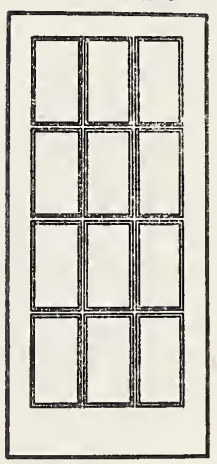

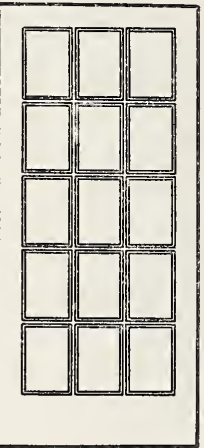

Sticking: Standard.

Approximate glass size for above layout

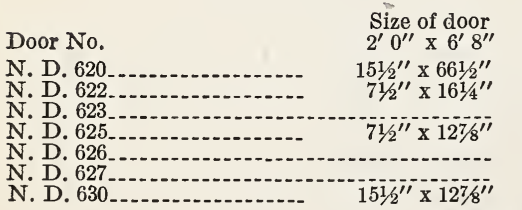

\begin{tabular}{|c|c|}
\hline Size of door & Size of door \\
\hline $2^{\prime} 6^{\prime \prime} \times 6^{\prime} 8^{\prime \prime}$ & $2^{\prime} 8^{\prime \prime} \times 6^{\prime} 8^{\prime \prime}$ \\
\hline $\begin{array}{l}211 / 2^{\prime \prime} \times 661 / 2^{\prime \prime} \\
101 / 2^{\prime \prime} \times 161 / 4^{\prime \prime}\end{array}$ & $\begin{array}{l}231 / 2^{\prime \prime} \times 661 / 2^{\prime \prime} \\
111^{\prime \prime} \times 11^{\prime \prime} 4^{\prime \prime}\end{array}$ \\
\hline $\begin{array}{r}101 / 2^{\prime \prime} \times 127 / 8^{\prime \prime} \\
6^{13} / 16^{\prime \prime} \times 161 / 4^{\prime \prime} \\
6^{13} / 16 \times 12^{7} / 8^{\prime \prime} \\
211 / 2^{\prime \prime} \times 1278^{\prime \prime}\end{array}$ & $\begin{array}{r}111 / 2^{\prime \prime} \times 127 / 8 " \\
71 / 2^{\prime \prime} \times 161 / 4^{\prime \prime} \\
71 / 2^{\prime \prime} \times 127 / 8 " \\
231 / 2^{\prime \prime} \times 1278^{\prime \prime}\end{array}$ \\
\hline
\end{tabular}
Size of door $3^{\prime} 0^{\prime \prime} \times 6^{\prime} 8^{\prime \prime}$ $27^{\prime \prime} 1 / 2 \times 661 / 2 \prime \prime$ $131 / 2^{\prime \prime} \times 1614^{\prime \prime}$ $131 / 2^{\prime \prime} \times 127 / 8^{\prime \prime}$ $813 / 16^{\prime \prime} \times 1614^{\prime \prime}$ $813 / 16^{\prime \prime} \times 127 / 8^{\prime \prime}$ $813 / 16^{\prime \prime} \times 127 / 8 " \prime$
$271 / 2^{\prime \prime} \times 127 / 8^{\prime \prime}$

Beads for glass included.

* Corner Lights.

Above doors also supplied with $31 / 2$ " stiles and top rail when so specified. Above doors also supplied with $11 \frac{1}{2}$ " bottom rail when so specified

See table 1, page 7, for sizes available. 


\section{SIDE LIGHTS}

\section{S.L. 675}

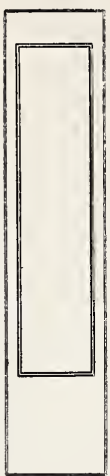

Stiles.

Top rail

Bottom rail.

Top and bottom rails made same width as in doors with which they are used.

Sticking: Standard.

\section{Beads for glass included.}

\section{S.L. 676}

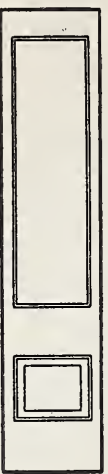

Stiles

Top rail

Bottom rail

Top and bottom rails made same width as in doors with which they are used.

Sticking: Standard.

Beads for glass included.

Bottom and lock rails can be reversed when so specified.

See table 1, page 6 , for sizes available.

\section{STORM DOORS}

N.D. 702

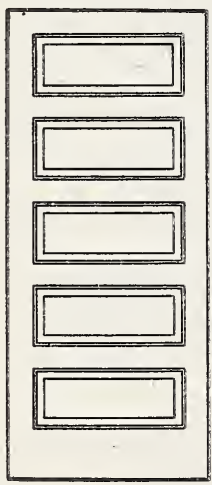

Stiles and top rail

Cross rails

Bottom rail

Raised panels 2 sides. Can also be furnished with 3-ply plywood flat panels, if desired. Sticking: Standard.
N.D. 703

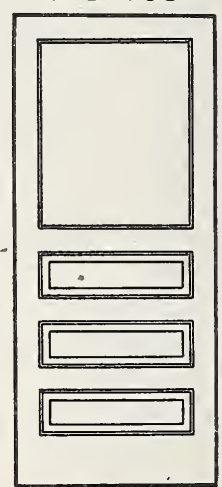

Stiles and top rail _.............. $434^{\prime \prime}$ Cross rails_.... Bottom rail .......... $95 / 8$

Raised panels 2 sides. Can also be furnished with 3-ply plywood flat panels, if desired. Sticking: Standard.

Size of door

Approx. glass size

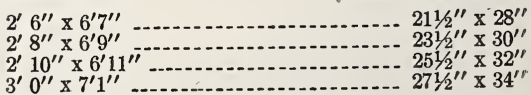

Beads for glass included.

See table 1, page 7, for sizes available. 


\section{CUPBOARD DOORS}

\section{N.D. 710}

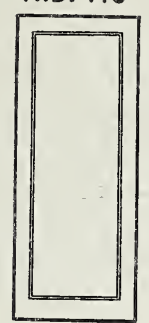

N.D. 711

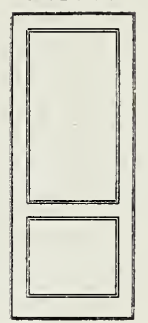

Stiles, top and cross rail $21 / 4^{\prime \prime}$ or $31 / 2^{\prime \prime}$

Stiles and top rail

$214^{\prime \prime}$ or $31 / 2 "$

Bottom rail

3-ply plywood flat panel. Sticking: Standard. Can also be furnished solid raised panel one side, flat one side.

Bottom rail........................ $314^{\prime \prime}$ or $41 / 2^{\prime \prime}$

3-ply plywood flat panels. Sticking: Standard. Can also be furnished solid raised panel one side, flat one side.

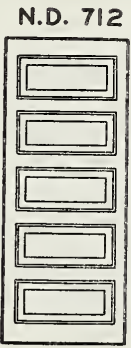

Stiles, top and cross rail............. $2 \frac{1}{1} 4^{\prime \prime}$ or $3 \frac{1}{2^{\prime \prime}}$ Bottom rail........................... $31 / 4^{\prime \prime}$ or $412^{\prime \prime}$

Raised panels. Sticking: Standard. Can also be furnished 3 -ply plywood flat panels, if desired.

N. D. 712 doors are made as follows:

Doors up to and including $2^{\prime} 0^{\prime \prime}$ high

Doors up to and including $2^{\prime \prime}$ high

Over $2^{\prime} 0^{\prime \prime}$ up to and including $3^{\prime} 0^{\prime \prime}$ high ...... 3 cross panels

Over $3^{\prime} 0^{\prime \prime}$ up to and including $4^{\prime} 0^{\prime \prime}$ high

Over $4^{\prime} 0^{\prime \prime}$ high............. 5 cross panels.

See table 1, page 6 , for sizes available 


\section{GARAGE DOORS}

N.D. 720

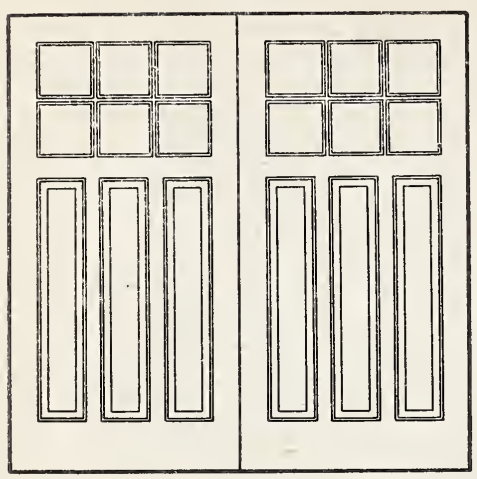

Stiles and top rail _... Cross rail

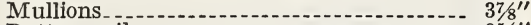
Bottom rail Vertical and horizontal bars between glass.- $1 / 2{ }^{\prime \prime}$

Raised panels 2 sides. Can also be furnished with 3-ply plywood flat panels, if desired. Stick. ing: Standard.

Beads for glass included.

\section{N.D. 722}

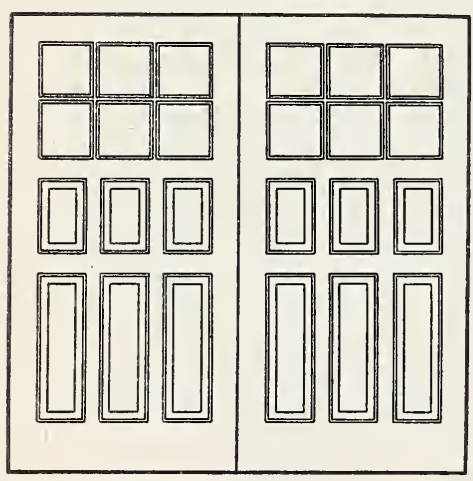

Stiles and top rail _

Cross rail Mullions......... $378^{\prime \prime}$ Bottom rail

Vertical and horizontal bars between glass_- $1 / 2^{\prime \prime}$

Raised panels 2 sides. Can also be furnished with 3-ply plywood flat panels, if desired. Sticking: Standard.

Beads for glass included.
N.D. 721

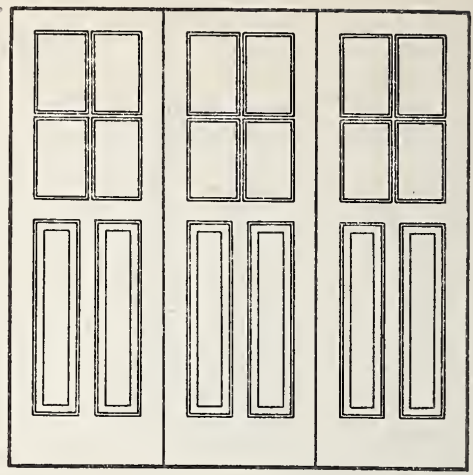

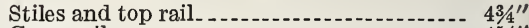
Cross rail Mullions Bottom rail Vertical and horizontal bars between glass.- $1 / 2^{\prime \prime}$

Raised panels. Can also be furnished with 3-ply plywood flat panels, if desired. Sticking: Standard.

Beads for glass included.

N.D. 723

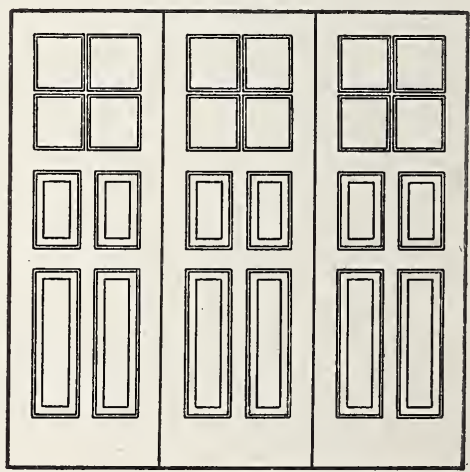

Stiles and top rail

Cross rail Mullions ......... $378^{\prime \prime}$ Bottom rail_............................. 95/8" Vertical and horizontal bars between glass_. $1 / 2^{\prime \prime}$

Raised panels 2 sides. Can also be furnished with 3-ply plywood flat panels, if desired. Sticking: Standard.

Beads for glass included.

See table 1, page 7, for sizes available. 


\section{GARAGE DOORS-Continued}

N.D. 724

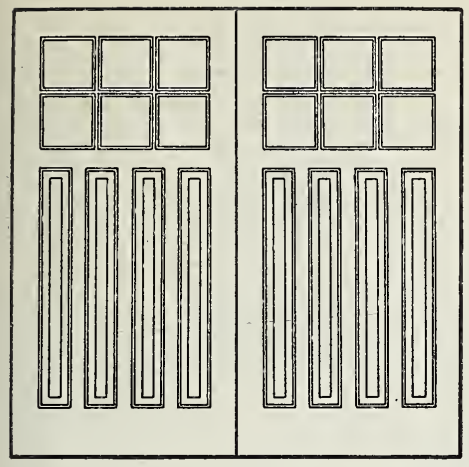

Stiles and top rail .

Cross rail

Mullions_._._.

Bottom rail .

Vertical and horizontal bars between glass_- $1 / 2^{\prime \prime}$

Raised panels 2 sides. Can also be furnished with 3-ply plywood flat panels, if desired. Sticking: Standard.

Beads for glașs included.
N.D. 725

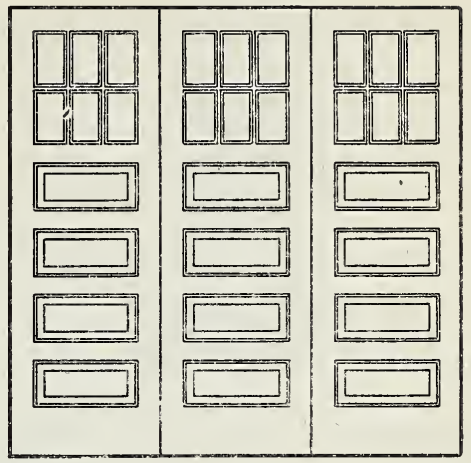

Stiles and top rail _..._._.

Cross rail

Bottom rail

Vertical and horizontal bars between glass.- $1 / 2^{\prime \prime}$

Raised panels 2 sides. Can also be furnished with 3-ply plywood flat panels, if desired. Sticking: Standard.

Beads for glass included.

\section{TOILET DOORS}
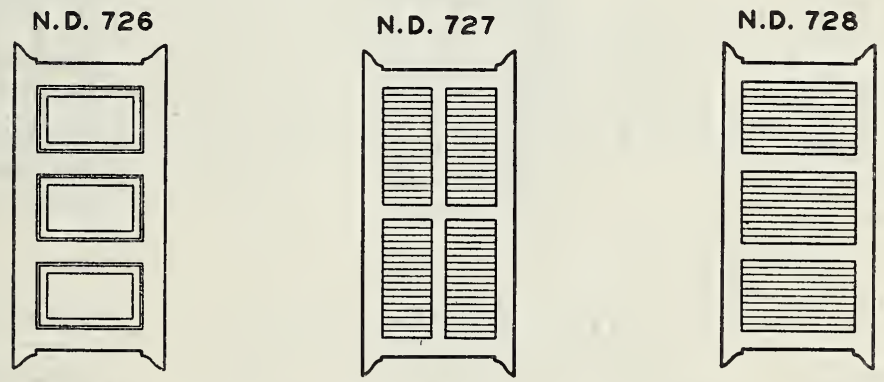

Stiles, top and bottom rail_._ $434^{\prime \prime}$ Stiles, top and bottom rail_. $31 \frac{1}{2 \prime} \quad$ Stiles, top and bottom rail_.- $31 / 2^{\prime \prime}$

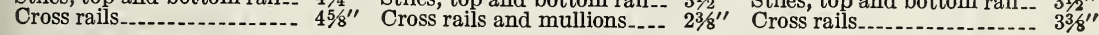

Raised panels 2 sides. Sticking: Standard.
Stationary slats. Sticking: Square.
Stationary slats. Sticking: Square.

Toilet doors can be supplied without lugs, if desired.

See table 1, page 7, for sizes available. 


\section{BLIND OR SUMMER DOORS}

N.D. 730

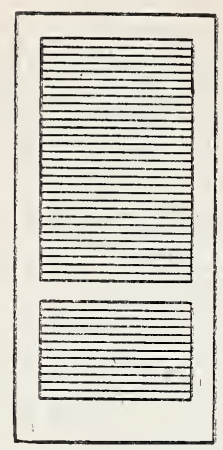

Stiles and top rail. ....................... $43 / 4^{\prime \prime}$ Cross rail Bottom rail ............................... $8^{\prime \prime}$ Stationary slats. Sticking: Square.
N.D. $73 !$

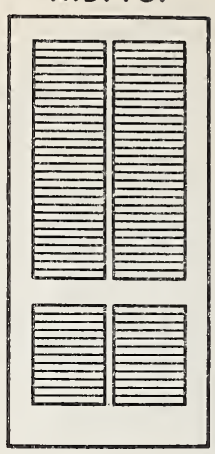

Stiles and top rail _... Cross rail ..... $53 / 4^{\prime \prime}$ Mullions .................. $23 / 8^{\prime \prime}$ Bottom rail. Stationary slats. Sticking: Square.

COMBINATION DOORS

N.D. 736

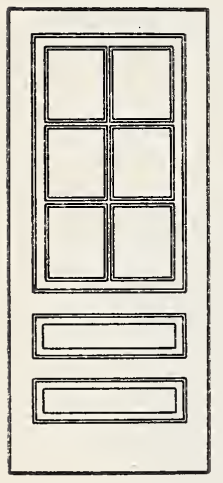

N.D. 742

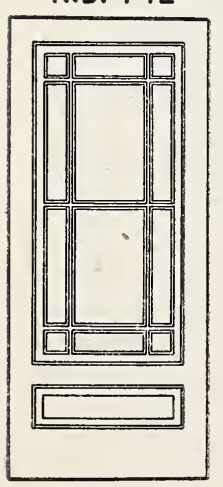

\section{N.D. 737}

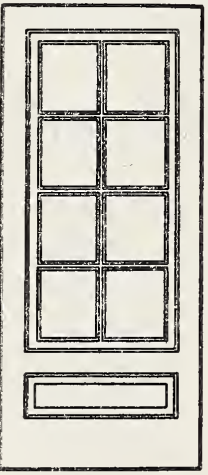

N.D. 749

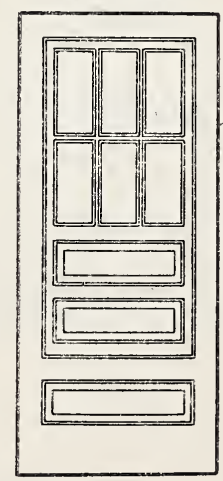

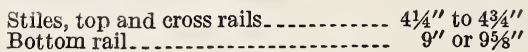

N.D. 739

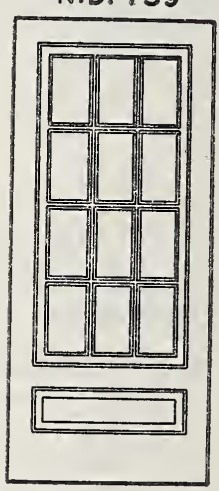

N.D. 756

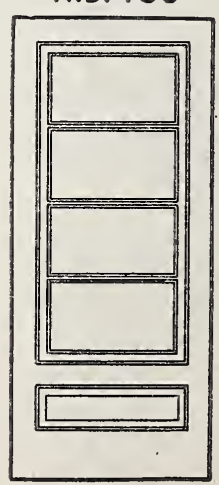

See table 1 , page 7 , for sizes available. 


\section{PONDEROSA PINE FLUSH DOORS}

25. All commercial standard ponderosa pine flush doors shall meet the following requirements:

26. Material.-Ponderosa pine used in the manufacture of flush doors shall be properly kiln-dried. A water-resistant glue shall be used.

27. Workmanship.-Flush doors shall be well manufactured and flat surfaces smoothly machine-sanded.

28. Construction. - The core of flush doors shall be formed of vertical blocks not over 2 inches wide on the face, securely glued together with water-resistant glue and with joints well staggered and the core surrounded with $3 / 4$-inch edge strip on all four edges. In lieu of vertical blocks, the core may be of stile, rail, and panel units, each unit made up of blocks, all of which when assembled with dowels, will make a solid core foundation for the cross banding and veneer. Cores shall be uniformly dried to proper moisture content and dressed to a smooth surface before applying the crossbanding. If crossbanded, the crossbanding and face veneers shall be glued to each side of the core and assembled under pressure. Exterior flush doors may be manufactured with wide edge strips to allow cutting down width and height of doors and they may also be made to permit cutting circle or other irregular top.

29. Veneers.-If cross-banded, the crossbanding shall be not less than $3 / 16$ inch or more than $1 / 8$ inch thick. Face veneers shall be from $1 / 16$ inch to $1 / 4$ inch thick before sanding, except where V-grooving is required, then $1 / 4$ inch thick before sanding.

30. Thickness. - Flush doors shall be $1 \frac{3}{4}$ inches thick and a thickness tolcrance of minus $1 / 16$ inch shall be allowed.

31. Grading.-Flush doors shall be of No. 1 grade. The stock shall be clear, except that bright sap, light-brown stain, and light-red kiln burn shall be permitted.
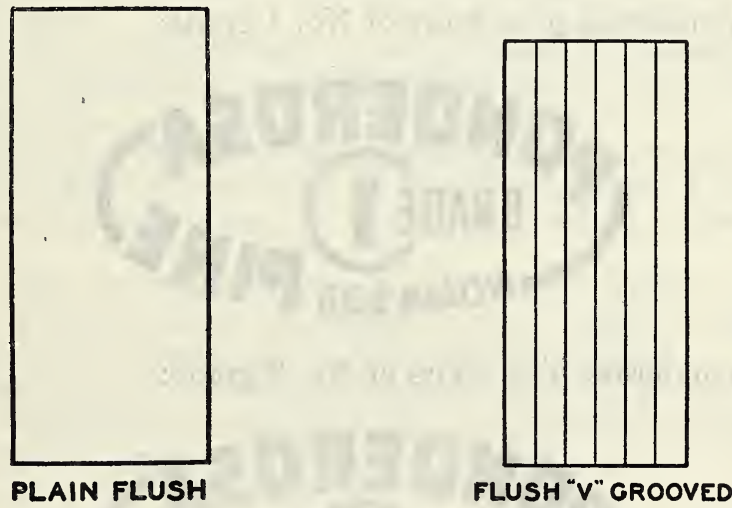

Light openings may be cut in these doors to suit the wishes of the purchaser.

See table 1, page 7 , for sizes available. 


\section{INSPECTION}

32. All ponderosa pine doors sold as conforming to the commercial standard are subject to inspection in the condition received, and complaints regarding any shipment shall be made within ten (10) days after receipt thereof. Any rejected doors shall be held, properly protected, for a period of thirty (30) days after notice of rejection and pending adjustment.

\section{LABELING}

33. In order to assure the purchaser that he is getting ponderosa pine doors of the quality specified, producers may individually, or in concert with their trade associations, issue guarantees, or grade mark each door by stamp, brand, or label as conforming to this standard. The following wording is recommended for the label:

This GRADE ........ ponderosa pine door is quaranteed by the manufacturer to conform to Commercial Standard CS120-46, as issued by the National Bureau of Standards of the U. S. Department of Commerce.

34. Grade Marking.

(Name of Manufacturer)

34a. The following grade-marking rules have been adopted by the National Door Manufacturers Association, Inc., as a means of assuring consumers and distributors that ponderosa pine doors conform to the high standards of quality defined herein.

$34 \mathrm{~b}$. Consumers and distributors may request that ponderosa pine doors be grade-marked. All ponderosa pine doors guaranteed to conform to the commercial grade rules as set forth herein may be stamped, labeled, or branded with the letters "NDMA", the grade designation, and an identification of the manufacturer by numerals.

34c. The following official grade designations have been approved by the National Door Manufacturers Association:

(1) For ponderosa pine doors of No. 1 grade:

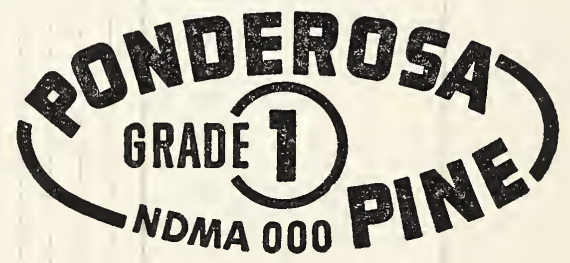

(2) For ponderosa pine doors of No. 2 grade:

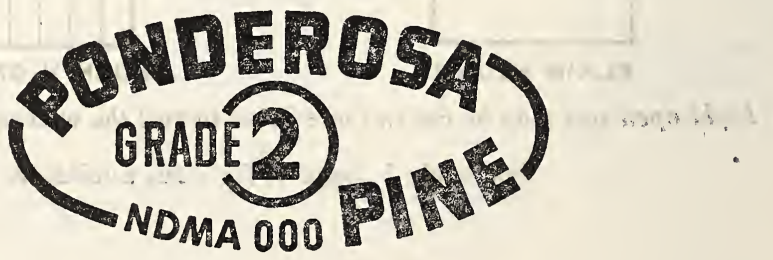


(3) For ponderosa pine doors of "Millrun" grade:

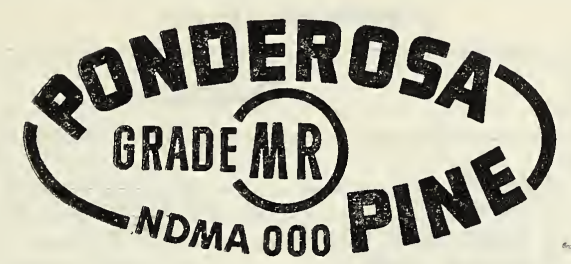

\section{NOMENCLATURE AND DEFINITIONS}

The definitions below give the meaning of various terms used in this standard:

Bars.-Wood divisions separating lights of glass.

Coped.-The shaping of the ends of rails, mullions, muntins, or bars so that they will cover and fit the contour of the sticking.

Core.-The innermost layer in veneered door construction.

Crossbanding. - The veneer which may be used in the construction of flush doors, which is placed between the core and face'veneers with the direction of the grain at right angles to that of the face veneer.

Flush door.-Made up of a core, crossbanding, and face veneers, or core and face veneers only.

Panel door.-Made up of stiles, rails, and one or more panels, the stiles and rails forming the frame around the panel.

Sash door.-Same as panel door, except one or more panels are replaced by glass.

Kiln dried.-Dried in a closed chamber in which the removal of moisture is controlled by artificial heat and usually by relative humidity.

Mullion.-An upright or vertical, bar in a door.

Muntin.-Any short or light bar, either vertical or horizontal, in a door between glass or panels and not extending the full width or length of the door.

Plywood panel.-A panel made up of core and face veneer.

$P$ itch seam.-An opening or imperfection parallel to the grain which is filled with pitch.

Rails. - The cross, or horizontal, pieces of the framework of a door. Bottom rail. - The bottom cross, or horizontal, piece of a door.

Lock rail.-The wide cross, or horizontal, rail of a door at lock height.

Top rail.-The top cross, or horizontal, piece of a door.

Sticking.-A mold which is worked on the edges of stiles, rails, mullions, muntins, or bars, adjacent to panels or glass.

Stiles. - The upright, or vertical, outside pieces of a door.

Veneered. - Made up of core and face veneers (may include crossbanding in flush doors.) 


\section{USE CLASSIFICATION INDEX}

\section{INTERIOR DOORS}

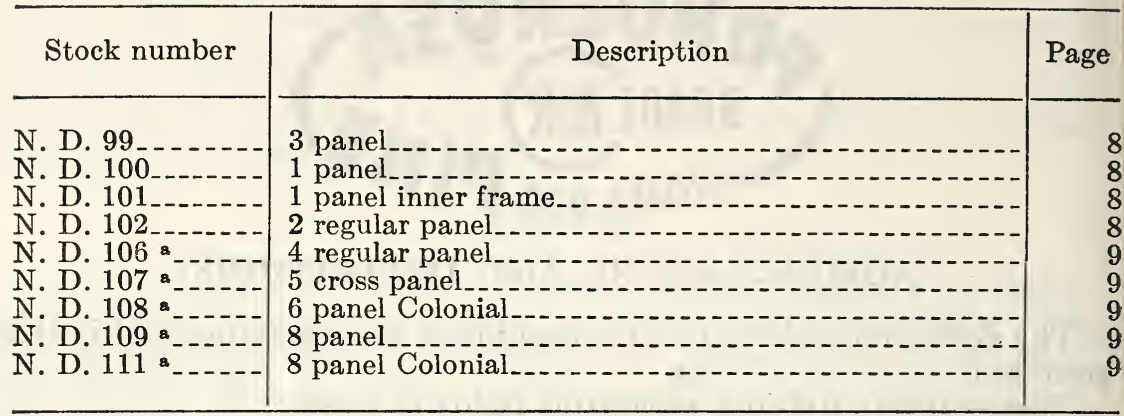

EXTERIOR Doors

N. D. 110

N. D. 112

N. D. 500

N. D. 501

N. D. 502

N. D. 505

N. D. 506

N. D. 507

N. D. 508

N. D. 509

N. D. 510

N. D. 511

N. D. 512

N. D. 513

N. D. 514

N. D. 515

N. D. 516

N. D. 517

N. D. 519

N. D. 536

N. D. 537 .

N. D. 538

N. D. 539

N. D. 542

N. D. 549

N. D. 559

N. D. 560

N. D. 561

N. D. 562

N. D. 563

N. D. 567

N. D. 568

N. D. 569

N. D. 570

N. D. 575

N. D. 576

N. D. 578

N. D. 580

N. D. 582

N. D. 591

N. D. 592

N. D. 593

N. D. 594

\section{6 panel Colonial}

8 panel Colonial

1 panel \& 6 its., $2 \mathrm{w}$

1 panel \& 9 lts., $3 \mathrm{w}$

1 panel \& 12 lts., $3 \mathrm{w}$

1 panel \& 4 hor. lts

1 panel \& 1 lt.

1 panel \& 9 lts., 3 w

1 panel \& 8 lts., 2 w

1 panel \& 9 marg. Its

1 panel \& 12 lts., 3 w

2 hor. pan. \& 3 hor. Its

2 hor. pan. \& 1 lt.

2 hor. pan. \& 4 lts., $2 \mathrm{w}$

2 hor. pan. \& 6 lts., 3 w

2 hor. pan. \& 9 lts., $3 \mathrm{w}$

2 hor. pan. \& 3 vert. Its

3 hor. pan. \& 3 hor. Its

3 hor. pan. \& 1 lt.

3 hor. pan. \& 4 lts., $2 \mathrm{w}$

3 hor. pan. \& 6 lts., $3 \mathrm{w}$

3 hor. pan. \& 3 vert. Its

4 hor. pan. \& 1 lt

2 vert. pan. \& 1 lt

2 vert. pan. \& 4 lts., 2 w

2 vert. pan. \& 6 lts., $2 \mathrm{w}$

2 vert. pan. \& 9 lts., $3 \mathrm{w}$

2 vert. pan. \& 3 hor. Its.

3 panel \& $1 \mathrm{lt}$

3 panel \& 4 lts., $2 \mathrm{w}$

3 panel \& 6 lts., $3 \mathrm{w}$.

3 panel \& 9 lts., $3 \mathrm{w}$

1 panel \& 1 lt...

1 panel \& 6 lts., $2 \mathrm{w}$

1 panel \& 9 lts., $3 \mathrm{w}$.

1 panel \& 12 lts., $3 \mathrm{w}$

2 vert. pan. \& 1 lt.

2 vert. pan. \& 3 vert. 1 ts

2 vert. pan. \& 4 vert. Its

2 vert. pan. \& 6 lts., $3 \mathrm{w}$

- Also for exterior use. 
Exterior Doors-Continued

\begin{tabular}{l}
\hline Stock number \\
\hline N. D. 596 \\
N. D. 597 \\
N. D. 598 \\
N. D. 600 \\
N. D. 605 \\
N. D. 606 \\
N. D. 607 \\
N. D. 608 \\
N. D. 609 \\
N. D. 612 \\
N. D. 613 \\
N. D. 620 \\
N. D. $622 \mathrm{~b}$ \\
N. D. 623 \\
N. D. 625 \\
N. D. 626 \\
N. D. 627 \\
N. D. 630 \\
N. D. 635 \\
N. D. 636 \\
N. D. 637 \\
N. D. 638 \\
N. D. 640 \\
N. D. 641 \\
N. D. 642 \\
N. D. 644 \\
D
\end{tabular}

\begin{tabular}{|c|c|}
\hline tion & Page \\
\hline 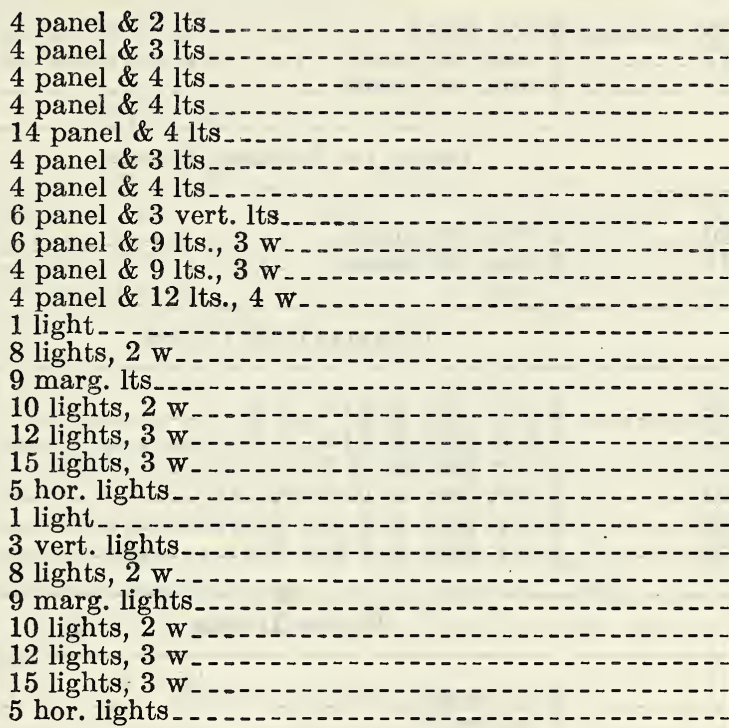 & $\begin{array}{l}17 \\
17 \\
18\end{array}$ \\
\hline
\end{tabular}

Sidelights

S. I. 675

S. T. 676 .
1 panel \& 1 light.

\section{Storm Doors}

N. D. 702

N. D. 703 .
5 hor. panel

3 hor. pan. \& 1 it.

\section{Cupboard Doors}

N. D. 710 .

N. D. 711 .

N. D. 712 .
1 panel.

2 hor. panel.

2 to 5 hor. panel
23

\section{Garage Doors}

N. D. 720

N. D. 721 .

N. D. 722

N. D. 723 .

N. D. 724 .

N. D. 725
3 vert. pan. \& 6 lts., 3 w

2 vert. pan. \& 4 lts., 2 w

6 pan. \& 6 lts. $3 \mathrm{w}$

4 pan. \& 4 lts., $2 \mathrm{w}$

4 vert. pan. \& 6 lts., $3 \mathrm{w}$

4 hor. pan. \& 6 lts., 3 w. 
ToILET Doors

\begin{tabular}{|c|c|c|}
\hline Stock number & Description & Page \\
\hline $\begin{array}{l}\text { N. D. } 726 \\
\text { N. D. } 727 \ldots \\
\text { N. D. } 728 \ldots\end{array}$ & $\begin{array}{l}3 \text { hor. panel } \\
4 \text { stat. slat panel } \\
3 \text { stat. slat panel }\end{array}$ & $\begin{array}{l}25 \\
25 \\
25\end{array}$ \\
\hline
\end{tabular}

Blind or Summer Doors

\begin{tabular}{|c|c|c|}
\hline $\begin{array}{l}\text { N. D. } 730 \\
\text { N. D. } 731 \ldots\end{array}$ & $\begin{array}{l}2 \text { stat. slat panel } \\
4 \text { stat. slat panel }\end{array}$ & $\begin{array}{l}26 \\
26\end{array}$ \\
\hline
\end{tabular}

Combination Doors

\begin{tabular}{|c|c|c|}
\hline $\begin{array}{l}\text { N. D. } 736 \\
\text { N. D. } 737-179-1 \\
\text { N. D. } 739 \\
\text { N. D. } 742 \\
\text { N. D. } 749 \\
\text { N. D. } 756\end{array}$ & $\begin{array}{l}2 \text { hor. pan. \& } 6 \text { lts., } 2 \text { w w } \\
1 \text { hor. pan. \& } 8 \text { lts., } 2 \text { w. w } \\
1 \text { hor. pan. \& } 12 \text { lts., } 3 \text { warg. } \\
1 \text { hor. pan. \& } 12 \text { marg. lts. } \\
3 \text { hor. pan. \& } 6 \text { lts., } 3 \text { w } \\
1 \text { hor. pan. \& } 4 \text { hor. } 1 \text { ts }\end{array}$ & $\begin{array}{l}26 \\
26 \\
26 \\
26 \\
26 \\
26\end{array}$ \\
\hline
\end{tabular}

FLUSH Doors

Flush .

Plain flush

\section{EFFECTIVE DATE}

35. The standard is effective for new production from October 1 , 1946.

\section{STANDING COMMITTEE}

36. The following individuals comprise the membership of the standing committee, which is to review, prior to circulation for acceptance, revisions proposed to keep the standard abreast of progress. Each organization nominated its own representative. Comment concerning the standard and suggestions for revision may be addressed to any member of the committee or to the Division of Trade Standards, National Bureau of Standards, which acts as secretary for the committee.

W. H. Schwa (chairman), Huttig Manufacturing Co., Muscatine, Iowa.

Glen Converse, Anson \& Gilkey Co., Merrill, Wis.

Lewis C. Paisley, Farley \& Loetscher Manufacturing Co., Dubuque, Iowa.

R. J. Lillibridge, National Door Manufacturers Association, Inc., 712 Transportation Bldg., Washington, D. C.

Frank Stevens, Ideal Co., Waco, Tex. (Representing Ponderosa Pine Woodwork).

Cla ud F. Wilson, Kimball \& Wilson, Inc., 2127 Fenkell Ave., Detroit 3, Mich. (Representing Woodwork Jobbers Service Bureau.)

Norman B. Cove, Hager \& Cove Lumber Co., Lansing, Mich. (Representing Michigan Retail Lumber Dealers Association.) 
W. A. Compton, Allen Millwork Manufacturing Co., Shreveport, La. (Representing Southern Sash \& Door Jobbers Association).

EDWARD A. Poynton, Director of Construction, Office of Indian Affairs, U. S. Department of the Interior, Chicago 54, Ill.

HAROLD A. PARKS, Hardware Manufacturers' Statistical Association, 205 Church St. (P. O. Box 1603), New Haven 6, Conn.

E. W. MACY, Property Standards Unit, Federal Housing Administration, National Housing Agency, Washington 25, D. C.

\section{HISTORY OF PROJECT}

37. On December 30, 1943, the National Door Manufacturers Association requested the cooperation of the National Bureau of Standards in the establishment of a commercial standard for standard stock ponderosa pine doors. A draft of the proposed standard was submitted on January 29,1944 , to producers, and to a number of technical, distributor, and consumer organizations for their views and comment. All comment was carefully considered at a meeting held in Chicago, Ill., on March 28, 1944 . The standard was then adjusted to represent the composite views of all interested groups, and circulated on May 31, 1944, to the trade for written acceptance. Upon receipt of official acceptance, estimated to represent a satisfactory majority of the production by volume, and in the absence of active valid opposition, the standard was promulgated on August 15, 1944, as Commercial Standard CS120-44, to become effective for new production on September 15, 1944.

\section{FIRST REVISION}

38. On April 2, 1946, the Chairman of the Standing Committee recommended the deletion of 18 outmoded or obsolete designs; the inclusion of 2 new designs that have become popular during the past two years and an improved listing of the standard sizes, segregated according to designs and use. On approval by the Standing Committee, this revision was circulated on July 17,1946 to the trade for written acceptance. Following acceptance by a satisfactory majority, the success of the revision was announced on August 30, 1946, as Commercial Standard CS120-46. 



\section{ACCEPTANCE OF COMMERCIAL STANDARD}

If acceptance has not previously been filed, this sheet properly filled in, signed, and returned will provide for the recording of your organization as an acceptor of this commercial standard.

\section{Date}

Division of Trade Standards,

National Bureau of Standards,

Washington 25, D. C.

Gentlemen:

We believe that the Commercial Standard CS120-46 constitutes a useful standard of practice, and we individually plan to utilize it as far as practicable in the

Production $^{1} \quad$ Distribution $^{1} \quad$ Purchase $^{1} \quad$ Testing $^{1}$

of standard stock ponderosa pine doors.

We reserve the right to depart from it as we deem advisable.

We understand, of course, that only those articles which actually comply with the standard in all respects can be identified or labeled as conforming thereto.

Signature of authorized officer

$$
\text { (In ink) }
$$

\section{(Kindly typewrite or print the following lines)}

Name and title of above officer

Organization

Street address

City, zone, and State

1 Underscore which one. Please see that separate acceptances are filed for all subsidiary companies and Underscore which one. Please see that separate acceptances are fisted related interests, trade associations affiliates which should be listed separately as acceptors. In the case of related interests, trade associations,
trade papers, etc., desiring to record their general support, the words "General support" should be added after the signature. 


\section{TO THE ACCEPTOR}

The following statements answer the usual questions arising in connection with the acceptance and its significance:

1. Enforcement.-Commercial standards are commodity specifications voluntarily established by mutual consent of those concerned. They present a common basis of understanding between the producer, distributor, and consumer and should not be confused with any plan of governmental regulation or control. The United States Department of Commerce has no regulatory power in the enforcement of their provisions, but since they represent the will of the interested groups as a whole, their provisions through usage soon become established as trade customs, and are made effective through incorporation into sales contracts by means of labels, invoices and the like.

2. The acceptor's responsibility.-The purpose of commercial standards is to establish for specific commodities, nationally recognized grades or consumer criteria and the benefits therefrom will be measurable in direct proportion to their general recognition and actual use. Instances will occur when it may be necessary to deviate from the standard and the signing of an acceptance does not preclude such departures; however, such signature indicates an intention to follow the commercial standard where practicable, in the production, distribution, or consumption of the article in question.

3. The Department's responsibility. - The major function performed by the Department of Commerce in the voluntary establishment of commercial standards on a Nation-wide basis is fourfold: first, to act as an unbiased coordinator to bring all interested parties together for the mutually satisfactory adjustment of trade standards; second, to supply such assistance and advice as past experience with similar programs may suggest; third, to canvass and record the extent of acceptance and adherence to the standard on the part of producers, distributors, and users; and fourth, after acceptance, to publish and promulgate the standard for the information and guidance of buyers and sellers of the commodity.

4. Announcement and promulgation.-When the standard has been endorsed by a satisfactory majority of production or consumption in the absence of active, valid opposition, the success of the project is announced. If, however, in the opinion of the standing committee or the Department of Commerce, the support of any standard is inadequate, the right is reserved to withhold promulgation and publication. 


\section{ACCEPTORS}

39. The organizations and individuals listed below have accepted these grading specifications as their standard of practice in the production, distribution, and use of standard stock ponderosa pine doors. Such endorsement does not signify that they may not find it necessary to deviate from the standard, nor that producers so listed guarantee all of their products in this field to conform with the requirements of this standard. Therefore, specific evidence of quality certification should be obtained where required.

\section{ASSOCIATIONS}

(General Support)

American Institute of Architects, Cincinnati, Chapter, Cincinnati, Ohio.

American Specification Institute, Chicago, $\mathrm{nl}$.

Building Officials Conference of America, Inc., Washington, D. C.

Carolina Lumber \& Building Supply Association, Charlotte, N. C.

Michigan Retail Lumber Dealers Association, Lansing, Mich.

Mississippi Retail Lumber Dealers Association, Inc., Jackson, Miss.

National Contract Hardware Association, New York, N. Y.

National Door Manufacturers Association, Chicago, Ill.

Prefabricated Home Manufacturers' Institute, Washington, D. C.

Souttern California Retail Lumber Association,

Los Angeles, Calif.

Southern Sash \& Door Jobbers Association, Memphis, Tenn.

Southern Woodwork Association, Atlanta, Ga.

\section{FIRMS}

Acme Sash \& Door Co., Cincinnati, Ohio.

Adams, Franklin O., Tampa, Fla.

Adams \& Kelly Co., Omaha, Nebr.

Adams-Rogers Co., Indianapolis, Ind.

Adkins \& Co., s. S., Salisbury, Md.

Akron Sash \& Door Co., The, Akron, Ohio.

Allen Millwork Manufacturing Co., Shreveport, La.

Allith Prouty, Inc., Danville, Ill.

Altfillisch, Charles, Decorah, Iowa.

Amarillo Sash \& Door Co., Houston, Tex.

Anderson \& Lind Manufacturing Co., Ohicago, Ill.

Andrews, Jones, Biscoe \& Goodell, Boston, Mass.

Andrews Lumber Co., C. E., New Bethlehem, Pa.

Anson \& Gilkey Co., Merrill, Wis.

Arizona Sash, Door \& Glass Co., Tucson, Ariz.

Asheim \& Wilkins, Bridgeport, Conn.

Ashton Co., C. J., Detroit, Mich.

Athens Lumber Co., A thens, Ga.

Aves Millwork Co., Inc., (formerly Arthur A. Aves,

Inc.), Yakima, Wash.

Babin Sash \& Door Co., The, Cleveland, Ohio.

Baltimore, City of, Department of Public Works, Division of Architecture, Baltimore, Md.

Barnes Lumber Co., W. F. \& J. F., Waco, Tex.

Baxter \& Co., C. B., Kansas City, Mo.

Beach Mill \& Supply Co., Miami Beach, Fla.

Beasley \& Sons Co., Nashville, Tenn.

Becker Danowitz Co., Inc., Brooklyn, N. Y.

Belli, Edo J., Chicago, Ill.

Bennett-Bailey Lumber Co., Minneapolis, Minn.

Bennett Lumber Corporation, N. Tonawanda, N. Y.

Beuttler, William, Sioux City, Iowa.

Bickford, Robert Turner, Elmira, N. Y.

Binswanger \& Co., Inc., Richmond, Va., and Columbia, S. C.

Bishop, Horatio W., La Mesa, Calif.

Blount Lumber Co., The, Lacona, N. $\mathbf{Y}$.

Boehm, George A., New York, N.'Y.

Boise Sash \& Door Factory, Boise, Idaho.

Bosman \& Casson, Inc., Harrison, N. J.

Brown-Graves Co., Akron, Ohio.

Bryan-Beck, Staunton, Va.

Buckley Door Co., F. S., San Francisco, Calif.

Bucky, Fred W., Jr., Jacksonville, Fla.

Buell Lumber \& Manufacturing Co., Dallas, Tex.

Buffalo, City of, Architectural Service, Department

of Public Works, Buffalo, N. Y.

Buffalo-Plywood Corp., Buffalo, N. Y.

Buffelin Lumber \& Manufacturing Co., Fort Worth, Tex.

Builders Supply Co., Bismarck, N. Dak.

Burritt Co., The A.'W., Bridgeport, Conn.

Byron Sash' \& Door Co., Inc., Louisville, Ky.

C \& M Construction Co., Inc., Philadelphia, Pa.

California Door Co., The, Los Angeles, Calif.

Cameron \& Co., Inc., Wm., Waco, Tex.

Cameron Lumber Co., Inc., Newburgh, N. Y.

Camlet, .J. Thomas, Passaic, N. J.

Cannon \& Mullen, Salt Lake City, Utah.

Carnahan Manufacturing Co., Loogootee, Ind.

Carr, Adams \& Collier Co., Dubuque, Iowa.

Carter-Lee Lumber Co., Indianapolis, Ind.

Cavalier Corp., Chattanooga, Tenn.

Cellar Lumber Co., Westerville, Ohio.

Central Glazing Co., Fort Worth, Tex.

Central Kentucky Supply Co., Inc., Lexington, Ky.

Central Wholesale Co., Inc., Shreveport, La.

Chapin, Rollin C., Minneapolis, Minn. (General support.)

Chapin Lumber Co., The, Aurora, Colo.

Charlottesville Lumber Co., Inc., Charlottesville, Va.

Chicago \& Riverdale Lumber Co., Chicago, Ill.

Cincinnati, City of, Cincinnati, Ohio.

Cleary Millwork Co., Inc., Ansonia, Conn.

Clem Lumber Co., Dallas, Tex.

Coffin, R. V., Seattle, Wash.

Collier-Barnett Co., The, Toledo, Ohio.

Combs Lumber Co., Inc., Lexington, Ky.

Coolidge, Shepley, Bulfinch \& Abbott, Boston, Mass.

Corbin, P. \& F. (Division of The American Hardware Corp.), New Britain, Conn. (General support)

Corddry Co., The, Snow Hill, Md.

Cram \& Ferguson, Boston, Mass.

Crawford Co., Inc., Baton Rouge, La.

Crawford Manufacturing Co., El Paso, Tex.

Cross, Austin, \& Ireland Lumber Co., Brooklyn, N. Y.

Crouch \& Beahan Co., Rochester, N. Y.

Crowell \& Lancaster, Bangor, Maine.

Cummings, George Bain, Binghamton, N. Y.

Curtis Co's., Inc., Clinton, Iowa, Minneapolis, Minn., and Topeka, Kans.

Curtis Co's., Inc., Chicago Division, Chicago, Ill.

Curtis Co's., Inc., Lincoln Division, Lincoln, Nebr.

Curtis Co's., Inc., Sioux City Division, Sioux City, Iowa.

Curtis Co's., Inc., Wausau Division, Wausau, Wis.

Curtis Co., Inc., Ros, Detroit, Mich.

Danville Lumber \& Manufacturing Co., The, Dan. ville, $\mathrm{Va}$.

Darby-Bogner \& Associates, Milwaukee, Wis.

Davidson Sash \& Door Co., Inc., Lake Charles, La., and Austin, Tex.

De Jarnette, Charles W., Des Moines, Iowa (General support).

Dcalers Wholesale Supply, Inc., Detroit, Mich.

Deats Sash \& Door Co., Los Angeles, Calif.

Deming \& Thompson Co., Inc., Frankfort, Ind.

Donlin Co., The, St. Cloud, Minn.

Donovan, John J., Berkeley, Calif.

Dykes Lumber Co., New York, N. Y.

Empire Millwork Corp., Corona, N. Y.

Esters Lumber Co., Birmingham, Ala.

Everett \& Associates, H. F., Allentown, $\mathrm{Pa}$.

Farley \& Loetscher Manufacturing Co., Dubuque, Iowa.

Field Detroit Co., Detroit, Mich.

Fink \& Schindler Co., Inc., San Francisco, Calif.

FitzGibbon, T. David, Norfolk, Va.

Flannagan, Eric G., Henderson, N. C.

Ford Lumber Co., Ivon R., McDondough, N. Y.

Fort Wayne Builders Supply Co., Fort Wayne, Ind. 
Foster Lumber Co., R. S., Indianapolis, Ind. Fuller \& Co., W. P., Boise, Idaho.

Furer, Wm. C., Honolulu, T. H.

Galliher \& Hugely, Inc., Washington, D. O.

General Millwork Corp., Utica, N. Y.

General Sash \& Door Co., Tulsa, Okla.

Gibson Door Co.. The, Utica, N. Y.

Glendale Sash \& Millwork Corp., Gilendale, Brooklyn, N.Y.

Glynn-Johnson Corp., Chicago, Ill.

Goshen Sash \& Door Co., Goshen, Ind.

Great Lakes Sash \& Door Co., The, Cleveland, Ohio.

Grcen Lumber Co., The, Laurel, Miss.

Greene \& Wood, Inc., New Bedford, Mass.

Gresham Lumber Co., Inc., Griffin, $\mathrm{Ga}$.

Grier Lumber Co., Cheyenne, Wyo.

Grimm Planing Mill, Albert C., Evansville, Ind.

Hacker-Sime Co., Joliet, Ill.

Hager-Cove Lumber Co., Lansing, Mich.

Hahn, Stanley W., Cleveland, Ohio.

Haley Bros., Santa Monica, Calif.

Hannaford \& Son, Samuel, Cincinnati, Ohio.

Haralson \& Mott, Fort Smith, Ark.

Harbor Plywood Corp., Chicago, Ill., and Jacksonville, Fla.

Harbor Salcs Co., The, Baltimore, Md., and Washington, D. C.

Hartung Co., F. L., Seattle, Wash

Hasness, Carlisie D̈., Harrisburg, $\mathrm{Pa}$.

Hass Wholesale, Inc., South Bend, Ind.

Haw kins Lumber \& Warehouse Co., Boston, Mass.

Helfensteller, Hirsch \& Watson, St. Louis, Mo.

Higgins, Charles H., New York, N. Y.

Hodgdon, Charles, San Gabriel, Calif. (General support).

Hoener, P. John, St. Lonis, Mo.

Hollenbek-Bush Planing Mill Ċo,, Fresno, Calif.

Holsman \& Fiolsman \& Klekamp, Chicago, Ill.

Home Building Corp., Sedalia, Mo.

Home Corporation of America, De Kalb, Ill.

Hope, Frank L., Jr., San Diego, Calif.

Houston Ready-Cut House Co., Houston, Tex.

Houston Sash \& Door Co., Houston, Tex.

Huttig Manufacturing Co., Muscatine, Iowa.

Huttig Sash \& Door Co., Louisville, Ky., and other cities.

Hyde-Murphy Co., Ridgway, $\mathrm{Pa}$

Illinois Lumber Manufacturing Co., Cairo, $\mathrm{nl}$.

Independent Lumber Co., The, Grand Junction, Colo.

Interstate Sash \& Door Co., The, Canton, Ohio.

Iron City Sash \& Door Co., Pittsburgh, Pa.

Iron Mountain, City of, Lumber Yard, Iron Mountain, Mich.

Ivey, Inc., Edwin J., Seattle, Wash.

Jacksonville Sash \& Door Co., Jacksonville, Fla.

Jersey Millwork Corp., Jersey City, N. J.

Johnson \& Wimsatt, Inc., Washington, D. C.

Johnstone, Harry Inge, Mobile, Ala.

Kaaz Woodwork Co., Inc., Leavenworth, Kans.

Keely Plywood Co., Hal, Pittsburgh, Pa.

Kilham, Hopkins \& Greeley, Boston, Mass.

Klinger Manufacturing Co., San Antonio, Tex.

Kyle, Herbert S., Charleston, W. Va. (General support).

Law, Law, Potter \& Nystrom, Madison, Wis.

Lefken, L. J., Cincinnati, Ohio.

Lentz Co., A., Wauwatosa, Wis.

Lew is Lumber Co., Spring Lake, N. J

Loeb, Laurence M., White Plains, N. Y.

Loetscher \& Burch Manufacturing Co., Des Moincs, Iowa.

Long-Bell Lumber Co., The, Kansas City, Mo.

Lumber \& Millwork Co. of Philadelphia, The, Philadelphia, $\mathrm{Pa}$.

Lumbermen's Credit \& Warehouse Co., Kalamazoo, Mich.

Lyman Hawkins Lumber Co., The, Akron, Ohio.

Lyon-Gray Lumber Co., Dallas, Tex.

M \& M Woodworking Čo., Portland, Oreg.

Mahoney Sash \& Door Co., The, Canton, Ohio.

Mann \& Co., Hutchinson, Kans.

Markland Contracting Co., M. B., Atlantic City, N. J.

Marquard Sash \& Door Manufacturing Co., The, Cleveland, Ohio.

Martin, Edgar, Chicago, Ill.

Martin Lumber Co., Springfield, Mass.
Mason City Millwork Co., Mason City, Iowa.

Mason \& Co., George D., Detroit, Mich.

McCallum, 1). D., Los Angeles, Calif.

McClelland Co., The, Davenport, Iowa.

McClung \& Co., C. M., Knoxville, Tenn.

McCoy \& Co., Inc., Lawrence R., Worcester, Mass.

McPhillips Manufacturing Co., Mobile, Ala.

Memphis Sash \& Door Co., Memphis, Tenn.

Merritt Lumber Yards, Inc., Reading, Pa.

Metropolitan Millwork Co., Brooklyn, N. Y.

Michigan Wholesalers, Inc., Jackson, Mich.

Midland Building Industries, Inc., Indianapolis, Ind.

Minot Builders Supply Co., Minot, N. Dak.

Montgomery \& Patteson, Charleston, W. Va.

Mooser, William, San Francisco, Calif.

Morgan Co., Oshkosh, Wis.

Morgan Millwork Co., The, Baltimore, Md.

Morrison-Merrill \& Co., Salt Lake City, Utah.

Mueller, Hair \& Hetterich, Hamilton, Ohio.

Muhlenberg Bros., Reading, $\mathrm{Pa}$.

Murphy \& Ames, Inc., Arlington, Va.

National Homes Corp., Lafayette, Ind.

Newton Lumber \& Manufacturing Co., The, Colorado Springs, Colo.

Nicolai Door Sales Co., San Francisco, Calif.

Nielsen Construction Co., Harrisonburg, Va.

Northern Sash \& Door Co., Hawkins, Wis.

Norwood Sash \& Door Manufacturing Co., Norwood, Cincinnati, Ohio.

Nurenburg, W. S., Fort Worth, Tex.

O \& N Lumber Co., Menomonie, Wis.

Oettinger Lumber CCo., Inc., Greensboro, N. O.

Officer, Gwynn, Lafayette, Calif.

Ohio City Sash \& Door Co., Davton, Ohio.

Olson Lumber Co., Alhambra, Calif.

Pacific Mutual Door Co., Chicago, Ill.

Paducah Sash \& Door Co., Inc., Paducah, Ky.

Patten-Blinn Lumber Co., Los Angeles, Calif.

Pease Woodwork Co., Inc., Cincinnati, Ohio.

Peek \& Sons, S. H., East Äurora, N. Y.

Pennsylvania, Commonwealth of, Property \& Supplies, Bureau of Stanỏards, Harrisburg, Pa.

Pepper, George W., Jr., Philadelphia, Pa.

Porter-Hadley Co., Grand Rapids, Mich.

Portsmouth Lumber Corporation, Portsmouth, Va.

Quigley Co., J. R., Gloucester City, N. J.

Racine Wood Products Co., Racine, Wis.

Radford Co., The, Duluth, Minn., and Oshkosh, Wis.

Radford \& Sanders, Inc., Baltimore, Md.

Ramsey \& Sons, Inc., A. H., Miami, Fla.

Reeb Millwork Corp., Roselle Park, N.J.

Resnikoff, Abraham, New York, N. Y.

Rinehimer Bros. Manufacturing Co., Elgin-Rockford, Ill.

Ritchie \& Associates, James H., Bnston, Mass.

Roach \& Musser Co., Muscatine, Iowa.

Robbins Door \& Sash Co., Scranton, Pa.

Roberson \& Son, Inc., A., Binghamton, N. Y.

Robert \& Co., Inc., Atlanta, Ga.

Roberts Corp., U. N., Davenport, Iowa.

Rock Island Sash \& Door Works, Rock Island, III.

Rockwell Bros. \& Co., Houston, Tex.

Rockwell Manufacturing Co., The, Randolph, Wis.

Rogers Lumber Co., The T. H., Oklahoma City, Okla.

Rounds \& Porter Co., Wichita, Kans.

Rudinger, Inc., C. R., South Kearny, N. J.

Ruggles Lumber Co., Carlos, Springfield, Mass.

St. Louis Sash \& Door Works, St. Louis, Mo.

Sash, Door \& Glass Corp., Richmond, Va.

Schulzke, William H., Moline, Ill.

Sears, Roebuck \& Co. Chicago, Ill.

Segelke \& Kohlhaus Co., La Crosse, Wis.

Semling-Menke Co., Merrill, Wis.

Seneca Lumber \& Millwork Co., The, Fostoria, Ohio.

Shenk Co, Henry, Erie, $\mathrm{Pa}$

Shutze \& Armistead, Atlanta, Ga.

Sibley Lumber Co., F. M., Detroit, Mich.

Simons, Inc., Minneapolis, Minn.

Sloan Lumber Co., Fort Worth, Tex.

Smith Co., Allen A., Toledo, Ohio.

Snedaker \& Co., Inc., Frank C., Philadelphia, Pa.

Snell Sash \& Door Co., St. Paul, Minn., and Omaha, Nebr.

Sothman Co., The, Grand Island, Nebr. 
Southern Counties Gas Co., Los Angeles, Calif. Southern Millwork \& Supply Co., Inc., Lafayette، La.

Southwestern Sash \& Door Co., Albuquerque, N. Mex.

Southwestern Sash \& Door Co., Joplin, Mo.

Specification Record, Chicago, Ill.

Spokane Woodworking Co., Spokane, Wash.

Standard Lumber Co., Pine Bluff, Ark.

Standard Lumber \& Supply Co., Fort Wayne, Ind. Standard Millwork \& Supply Co., Jackson, Miss. Staniey Works, The, New Britain, Conn.

Stark \& Co., Inc., Kansas City, Mo.

Staub \& Rather, Houston, Tex.

Steves Sash \& Door Co., San Antonio, Tex., and Corpus Christie, Tex.

Stoetzel, Ralph, Chicago, Ill.

Stokes \& Allyn, Portland, Oreg.

Sturtevant Millwork \& Lumber Corporation, Hicksville, Long Island, N. Y.

Summers Hardware \& Supply Co., Johnson City, Tenn.

Swan Lake Moulding Co., Klamath Falls, Oreg. Sweetwater Sash \& Door Co., Sweetwater, Tex.

Taylor, Ellery K., Haddonfield, N. J.

Temple, Seth J., Davenport, Iowa.

Texas Sash \& Door Co., Fort Worth, Tex.

Theiling-Lothman Manufacturing Co., St. Louis, Mo.

Thompson Lumber Co., Minneapolis, Minn.

Thorne, Henry Calder, Ithaca, N. Y.

Toombs \& Co., Springfield, Mo.

Townsend Sash, Door \& Lumber Co., Tampa, Fal.

Trexler Lumber Co., Allentown, $\mathrm{Pa}$.

Tulsa Rig, Reel \& Manufacturing Co., Tulsa, Okla. Tyson Mill \& Builders Supply Co., Orlando, Fla.

Underwood Coal \& Supply Co., Mobile, A la.

Union Planing Mill, Stockton, Calif.

Valdosta Builders Supply Co., Valdosta, $\mathrm{Ga}$.

Vaughan \& Sons, Geo. C., Houston, Tex., and San Antonio, Tex.

Velde Lumber Co., Pekin, Ill

Vetter Manufacturing Co., Stevens Point, Wis.
Virginia Polytechnic Institute, Blacksburg, Va. (General support.)

Wabash Screen Door Co., The, Chicago, $\mathrm{nl}$.

Wahlfeld Manufacturing Co., Peoria, Ill. (General support.)

Wanke Panel Co., Portland, Oreg.

Warren Bros., Co., Nash ville, Tenn.

Warren Lumber Co., The, Ft. Morgan, Colo.

Watertown Sash \& Door Co., Watertown, S. Dak.

Weinel Lumber Co., A. F., Columbia, Ill.

Welch, Carroll E., Huntington, N. Y.

West, Albert E., Boston, Mass.

Western Door \& Sash Co., Oakland, Calif.

Western Hardwood Lumber Co., Los Angeles, Calif.

Whissel Lumber Co., Inc., L. N., Buffalo, N. Y.

White Pine Sash Co. of Illinois, Chicago, Ill.

Whitmer Mills, Albuquerque, N. Mex.

Whittier Lumber \& Millwork Co., Newark, N. J.

Wholesale Building Supply, Inc., Oakland, Calif.

Williams \& Hunting Co., Cedar Rapids, Iowa.

Willson, Fred F., Bozeman, Mont.

Wilson \& Sons, Inc., W. A., Wheeling, W. Va.

Wimberly \& Thomas Hardware Co., Inc., Birming.

ham, Ala.

Wolverine Shingle \& Lumber Co., Detroit, Mich.

Wood Lumber Co., E. K., Los Angeles, Calif.

Zimmerman, A. C., Los Angeles, Calif.

\section{U. S. GOVERNMENT}

Agriculture, U. S. Department of, Washington, D. C.

Federal Works Agency, Public Buildings Administration, Washington, D. C.

Intcrior, U. S. Department of the, Office of Indian Affairs, Chicago, Ill.

National Housing Agency, Federal Housing Admin istration, Washington, D. C. (General support.)

National Housing Agency, Federal Public Housing Authority, Technical Division, Washington, D. C.

Justice, U. S. Department of, Bureau of Prisons, Washington, D. C.

6th Naval District, Naval Base, S. C.

\section{COMMERCIAL STANDARDS}

OS No.

\section{Item}

0-40. Commercial standards and their value to business (third edition)

1-42. Clinical thermometers (third edition).

2-30. Mopsticks.

3-40. Stoddard solvent (third edition).

4-29. Staple porcelain (all-clay) plumbing fixtures.

5-46. Pipe nipples; brass, copper, steel, and wrought-iron (second edition).

6-31. Wrought-iron pipe nipples (second edition). Superseded by CS5-46.

7-29. Standard weight malleable iron or steel screwed unions.

8-41. Gage blanks (third edition).

9-33. Builders' template hardware (second edition).

10-29. Brass pipe nipples. Superseded by CS5-46.

11-41. Moisture regains of cotton yarns (second edition).

12-40. Fuel oils (fifth edition).

13-44. Dress patterns (fourth edition).

14-43. Boys' button-on waists, shirts, junior and sport shirts (made from woven fabrics) (third edition).

15-46. Men's pajama sizes (woven fabrics) (third edition).

16-29. Wall paper.

17-42. Diamond core drill fittings (third edition).

18-29. Hickory golf shafts.

19-32. Foundry patterns of wood (second edition).

20-42. Staple vitreous china plumbing fixtures (third edition)

21-39. Interchangeable ground-glass joints, stopcocks, and stoppers (fourth edition).
CS No.

Item

22-40. Builders' hardware (nontemplate) (second edition).

23-30. Feldspar.

24-43. Screw threads and tap-drill sizes.

25-30. Special screw threads. Superseded by CS24-43.

26-30. Aromatic red cedar closet lining.

27-36. Mirrors (second edition).

28-46. Cotton fabric tents, tarpaulins and covers (second edition).

29-31. Staple seats for water-closet bowls.

30-31. Colors for sanitary ware.

31-38. Wood shingles (fourth edition).

32-31. Cotton cloth for rubber and pyroxylin coating.

33-43. Knit underwear (exclusive of rayon) (second edition)

34-31. Bag, case, and strap leather.

35-42. Plywood (hardwood and eastern red cedar) (second edition).

36-33. Fourdrinier wire cloth (second edition). 37-31. Steel bone plates and screws.

38-32. Hospital rubber sheeting.

39-37. Wool and part-wool blankets (second edition). (Withdrawn as commercis standard, July 14, 1941.)

40-32. Surgeons' rubber gloves.

41-32. Surgeons' latex gloves.

42-43. Structural fiber insulating board (third edition).

43-32. Grading of sulphonated oils.

44-32. Apple wraps.

45-45. Douglas fir plywood (sixth edition).

46-40. Hosierylengths and sizes (third edition).

47-34. Marking of gold-filled and rolled-goldplate articles other than watchcases.

48-40. Domestic burners for Pennsylvania anthracite (underfeed type) (second edition). 
CS No.

Item

49-34. Chip board, laminated chin board, and miscellaneous boards for bookbinding purposes.

50-34. Binders hoard for bookbinding and other purposes.

51-35. Marking articles made of silver in com. bination with gold.

52-35. Mohair pile fabrics (100-percent mohair plain velvet, 100 -percent mohair plain frieze, and 50-percent mohair plain frieze).

53-35. Colors and finishes for cast stone.

54-35. Mattresses for hospitals.

55-35. Mattresses for institutions.

56-41. Oak flooring (second edition).

57-40. Book cloths, buckrams, and impregnated fabrics for bookbinding purposes except library bindings (second edition).

58-36. Woven elastic fabrics for use in overalls (overall elastic webbing).

59-44. Textiles-testing and reporting (fourth edition).

60-36. Hardwood dimension lumber.

61-37. Wood-slat venetian hlinds.

62-38. Colors for kitchen accessories.

63-38. Colors for bathroom accessories.

64-37. Walnut veneers.

65-43. Methods of analysis and of reporting fiber composition of textile products (second edition).

66-38. Marking of articles made wholly or in nart of platinum.

67-38. Marking articles made of karat gold.

68-38. Liquid hypochlorite disinfectant, deodorant, and germicide.

69-38. Pine oil disinfectant.

70-41. Phenolic disinfectant (emulsifying type) (second edition) (published with CS71-41).

71-41. Phenolic disinfectant (soluble type) (second edition) (published with CS70-41).

72-38. Household insecticide (liquid spray type).

73-45. Old growth Douglas fir standard stock doors (third edition).

74-39. Solid hardwood wall paneling.

75-42. Automatic mechanical draft oil burners designed for domestic installations (second edition).

76-39. Hardwood interior trim and molding.

77-40. Sanitary cast-iron enameled ware.

78-40. Ground-and-polished lenses for sun glasses (second edition) (published with CS79-40).

79-40. Blown, drawn, and dropped lenses for sun glasses (second edition) (published with CS78-40).

80-41. Electric direction signal systems other than semaphore type for commercia and other vehicles subject to special motor vehicle laws (after market).

81-41. Adverse-weather lamps for vehicles (after market).

82-41. Inner-controlled spotlamps for vehicles (after market).

83-41. Clearance, marker, and identification lamps for vehicles (after market).

84-41. Electric tail lamps for vehicles (after market).

85-41. Electric license-plate lamps for vehicles (after market).

86.41. Electric stop lamps for vehicles (after market).

87-41. Red electric warning lanterns.

88-41. Liquid-burning flares.

89-40. Hardwood stair treads and risers.

90- . (Reserved for power shovels and cranes.)

91-41. Factory-fitted Douglas fir entrance doors.
CS No.

Item

92-41. Cedar, cypress and redwood tank stock lumber.

93-41. Portable electric drills (exclusive of high frequency).

94-41. Calking lead.

95-41. Lead pipe.

96-41. Lead traps and bends.

97-42. Electric supplementary driving and passing lamps for vehicles (after market).

98-42. Artists' oil paints.

99-42. Gas floor furnaces-gravity circulating type.

100-44. Porcelain-enameled steel utensils (second edition)

101-43. Flue-connected oil-burning space heaters equipped with vaporizing pot-type burners.

102- - (Reserved for Diesel and fuel-oil engines.)

103-42. Cotton and rayon velour (jacquard and

104-46. Wain). vaporizing pot-type oil burners (second edition).

105-43. Mineral wool; loose granulated, or felted form, in low-temperature in. stallations.

106-44. Boys' pajama sizes (woven fabrics) (second edition).

107-45. Commercial electric-refrigeration condensing units (second edition).

108-43. Treading automobile and truck tires.

109-44. Solid-fuel-burning forced-air furnaces.

110-43. Tire repairs-vulcanized (passenger, truck, and bus tires).

111-43. Earthenware (vitreous-glazed) plumbing fixtures.

112-43. Homogeneous fiber wallboard.

113-44. Oil-burning floor furnaces equipped with vaporizing pot-type burners.

114-43. Hospital sheeting for mattress protection.

115-44. Porcelain-enameled tanks for domestic use.

116-44. Bituminized-fibre drain and sewer pipe.

117-44. Mineral wool; blankets, blncks, insulat. ing cement, and pipe insulation for heated industrial equipment.

118-44. Marking of jewelry and novelties of silver.

(E)119-45.1 Dial indicators (for linear measurements).

120-46. Standard stock ponderosa pine doors (second edition).

121-45. Women's slip sizes (woven fabrics).

122-45. Western hemlock plywood.

123-45. Grading of diamond powder.

(E) 124-45.1 Master disks.

125-45. Prefabricated homes.

126-45. Tank mounted air compressors.

127-45. Self-contained mechanically refrigerated drinking water coolers.

128-45. Men's sport shirt sizes-woven fabrics (other than those marked with regular neckband sizes).

129-46. Materials for safety wearing apparel.

130-46. Color materials for art education in schools.

131-46. Industrial mineral wool products, all types - testing and reporting.

132-46. Hardware cloth.

133-46. Woven wire netting.

134-46. Cast aluminum cooking utensils (metal composition).

135-46. Men's shirt sizes (exclusive of work shirts).

136-46. Blankets for hospitals (wool, and wool and cotton).

137-46. Size measurements for men's and boy's shorts (woven fabrics).

Notice.-Those interested in commercial standards with a view toward accepting them as a basis of everyday practice may secure copies of the above standards, while the supply lasts, by addressing the Division of Trade Standards, National Bureau of Standards, Washington 25, D. C.

1 Where "(E)" precedes the CS number, it indicates an emergency commercial standard, drafted under war conditions with a view toward early revision. 